\title{
Numerical study of pressure and velocity fluctuations in nearly isotropic turbulence
}

\author{
By U. SCHUMANN \\ Institut für Reaktorentwicklung, Kernforschungszentrum Karlsruhe, \\ 7500 Karlsruhe, Postfach 3640, West Germany \\ AND G.S. PATTERSON \\ National Center for Atmospheric Research, Boulder, Colorado 80307
}

(Received 19 May 1975 and in revised form 8 January 1978)

The spectral method of Orszag \& Patterson has been extended to calculate the static. pressure fluctuations in incompressible homogeneous decaying turbulence at Reynolds numbers $R e_{\lambda} \lesssim 35$. In real space $32^{3}$ points are treated. Several cases starting from different isotropic initial conditions have been studied. Some departure from isotropy exists owing to the small number of modes at small wavenumbers. Root-mean-square pressure fluctuations, pressure gradients and integral length scales have been evaluated. The results agree rather well with predictions based on velocity statistics and on the assumption of normality. The normality assumption has been tested extensively for the simulated fields and found to be approximately valid as far as fourth-order velocity correlations are concerned. In addition, a model for the dissipation tensor has been proposed. The application of the present method to the study of the return of axisymmetric turbulence to isotropy is described in the companion paper.

\section{Introduction}

Fluctuations of the static pressure in incompressible, homogeneous, decaying turbulence are studied numerically by means of the Galerkin (spectral) method of Orszag (1971). This method has been implemented by Orszag \& Patterson $(1972 a, b)$ and has been proved to give reliable results for decaying isotropic turbulence at Reynolds numbers $R e_{\lambda} \lesssim 35$. For the present purpose this method has been extended to calculate the pressure field, which was not computed in the older version. This paper describes the method and its application to isotropic turbulence. Results for anisotropic homogeneous turbulence are described in the companion paper. Pressure fluctuations are of interest with respect to sound generation (Ribner 1964), turbulent diffusion (Taylor 1935), structural vibrations (Kadlec \& Appelt 1970) and momentum and kinetic energy transport (Rotta 1951).

Experimental investigations of the pressure fluctuations are rare. Most of them are confined to measurements of these fluctuations at a wall boundary or about sea waves. The relevant literature has been reviewed by Willmarth (1975). Pressure fluctuations away from boundaries have been studied in the wake of a cylinder (Kobashi 1957), in a mixing layer (Spencer \& Jones 1971), in jet flows (Arndt \& Nilsen 1971; Fuchs 1972; Hammersley \& Jones 1975) and in the lower atmospheric boundary layer (Elliot 1972). Apparently, no direct measurements exist for homogeneous turbulence. 
Theories which predict turbulent pressure fluctuations if the statistical properties of the velocity field are known have been developed for homogeneous and isotropic turbulence (Taylor 1935; Heisenberg 1948; Batchelor 1951, 1959; Limber 1951; Uberoi 1953, 1954; Ogura \& Miyakoda 1954; Kraichnan 1956a), for anisotropic and shear flow (Kraichnan 1956a,b; Ribner 1964) and for wall pressure fluctuations (Panton \& Linebarger 1974).

Direct numerical simulations of turbulent pressure fluctuations are reported by Deardorff (1970) and Schumann (1975 a) for channel flows and by Deardorff (1974) for the atmospheric boundary layer. Wall pressure fluctuations have been studied numerically by Schumann (1975b). All these are finite-difference simulations of high Reynolds number flows which require some subgrid-scale (SGS) model for that part of the turbulent flow not resolvable by the finite-difference grid.

The spectral method allows us to perform a numerical experiment for homogeneous turbulence in which it is simple to ' measure' pressure fluctuations. The present scheme strictly integrates a discretized version of the Navier-Strokes equations without any SGS modelling. Owing to the finite capacity of our computers, the range of scales which can be resolved is restricted. In the present scheme the ratio of the largest and smallest resolved scales is 16 . Consequently, we are restricted to rather low Reynolds numbers (approximately $R e_{\lambda}<40$ ) where we can hope to resolve at least all important scales of turbulence, e.g. from the integral length scale down to the Kolmogorov microscale. The Reynolds number attainable is, however, comparable with those found in classical wind-tunnel experiments, e.g. that of Uberoi (1953, 1954).

After a short description of the numerical method, the general appearance of the flow fields is described and the limitations of the present method are shown. Then some empirical relationships between the pressure and velocity statistics are checked and the normality of fourth-order velocity correlations is investigated. Finally, some empirical models for the kinetic energy dissipation rate and the dissipation tensor are deduced.

\section{Spectral pressure calculation}

The numerical procedure for the velocity field and its statistics has been discussed by Orszag \& Patterson $(1972 a, b)$ and by Riley \& Patterson (1974). The turbulent motion is assumed to satisfy the Navier-Stokes equations for an incompressible flow:

$$
\begin{gathered}
\partial \mathbf{u} / \partial t-\nu \nabla^{2} \mathbf{u}=\mathbf{s}-\nabla(p+E), \\
\nabla \cdot \mathbf{u}=0,
\end{gathered}
$$

where $\mathbf{u}=\mathbf{u}(\mathbf{x}, t)$ and $p=p(\mathbf{x}, t)$ are the velocity and kinematic pressure fields, respectively, as functions of the real space variable $\mathbf{x}$ and time $t$, and

$$
\boldsymbol{\omega} \equiv \nabla \times \mathbf{u}, \quad \mathbf{s}=\mathbf{u} \times \boldsymbol{\omega}, \quad E=\frac{1}{2} \mathbf{u} . \mathbf{u}
$$

are the vorticity, the velocity-vorticity cross-product and the kinetic energy. With periodic boundary conditions at the surfaces of a cubic box with side $L$, the velocity field (for example) may be expanded in a Fourier series.

$$
\mathbf{u}(\mathbf{x}, t)=\sum_{\text {all } \mathbf{k}} \hat{\mathbf{u}}(\mathbf{k}, t) \exp (i \mathbf{i} \mathbf{x} \cdot \mathbf{x}),
$$


where $\mathbf{k}=(2 \pi / L) \mathbf{n}$. For a numerical calculation, the integer vector $\mathbf{n}$ is restricted to the finite set $-\frac{1}{2} N \leqslant n_{i} \leqslant \frac{1}{2} N(i=1,2,3)$, where $N^{3}$ corresponds to the number of points treated in real space. The pressure is eliminated by twice taking the curl of (1). After the Fourier transformation, we have, in wavenumber space,

$$
d \hat{\mathbf{a}} / d t+\nu k^{2} \hat{\mathbf{u}}=\hat{\boldsymbol{\tau}}, \quad \mathbf{k} \cdot \hat{\mathbf{u}}=0, \quad \hat{\boldsymbol{\tau}}=-k^{-2}[\mathbf{k} \times(\mathbf{k} \times \hat{\mathbf{s}})],
$$

where $\hat{\mathbf{s}}=\hat{\mathbf{s}}(\mathbf{k}, t)$ is the Fourier transform of $s(\mathbf{x}, t)$ and $\boldsymbol{\omega}(x, t)$ is the inverse Fourier transform of $i \mathbf{k} \times \hat{\mathbf{a}}(\mathbf{k}, t)$. The Fourier transforms are computed by using the fast Fourier transform and eliminating the aliasing interactions in $s$ as described by Patterson \& Orszag (1971).

To compute the pressure itself, we proceed as follows. Taking the divergence of (1) gives

$$
0=-\nabla \cdot s-\nabla^{2}(p+E)
$$

and after Fourier transforming we have

$$
\hat{p}=-i k^{-2} \mathbf{k} \cdot \hat{\mathbf{s}}-\hat{E},
$$

where $\widehat{E}=\widehat{E}(\mathbf{k}, t)$ is the Fourier transform of $E(\mathbf{x}, t)$. The real pressure $p(\mathrm{x}, t)$ is computed by inverse Fourier transformation of $\hat{p}=\hat{p}(\mathbf{k}, t)$. The amount of computer time and storage is increased by a factor of $\frac{4}{3}$ if the pressure field is calculated in addition to the velocity and vorticity fields. The method has been checked against pressure fields computed for random velocity fields from the convolution sum (Orszag \& Kruskal 1968):

$$
\hat{p}(\mathbf{k}, t)=-\frac{1}{k^{2}} \sum_{\mathbf{k}^{\prime}+\mathbf{k}^{\prime \prime}=\mathbf{k}}\left[\mathbf{k}^{\prime \prime} \cdot \hat{\mathbf{u}}\left(\mathbf{k}^{\prime}\right)\right]\left[\mathbf{k} \cdot \hat{\mathbf{u}}\left(\mathbf{k}^{\prime \prime}\right)\right] .
$$

For the present computations, $N=32$ and $L=\pi$ (in arbitrary units). Starting from some random initial values, we step the velocity field forward in finite time steps $\Delta t$, using the leapfrog scheme and treating the viscous terms implicitly.

\section{Isotropic homogeneous turbulence simulations}

\subsection{Run specification and general description}

Four cases, labelled $I 1-I 4$, have been run for isotropic turbulence. Initially, the Fourier coefficients for the velocity field were chosen to be independent, Gaussianly distributed random variables with a prescribed isotropic energy spectrum $\widehat{E}(k, t=0)$. As stated in the introduction, the present scheme is limited to moderately high Reynolds numbers. In addition, in order to limit truncation errors, we must require that the energy spectrum decreases sufficiently fast near the upper and lower wavenumber cut-offs used in the numerical simulation. Initial values with an energy spectrum of the form

$$
\widehat{E}(k, 0)=16(2 / \pi)^{\frac{1}{2}} v_{0}^{2} k_{\text {peak }}^{-5} k^{4} \exp \left[-2\left(k / k_{\text {peak }}\right)^{2}\right]
$$

are suitable in this respect. Such a spectrum is typical for the final period of decay when the nonlinear energy transfer is small in comparison with the viscous dissipation (Batchelor \& Townsend 1948). However, for $R e_{\lambda} \sim 30$, we must expect strong energy transfer to high wavenumbers. This results in a less rapid decrease in the spectrum at high wavenumbers. The spectral form (9) implies a decay of the kinetic energy as $t^{-n}$, where $t$ is the time and $n$ is of the order of $2 \cdot 5$. 


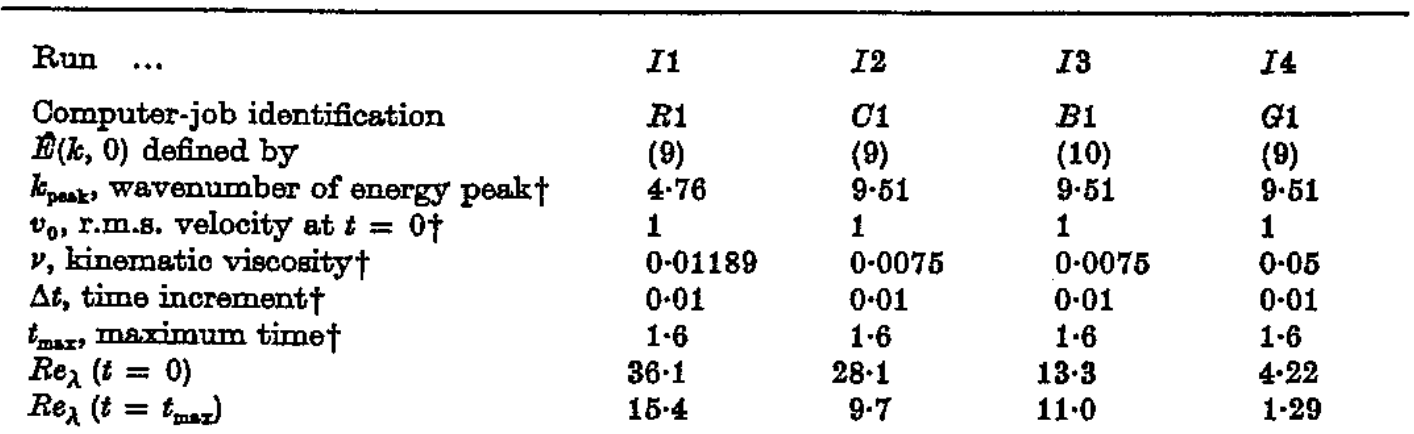

$\dagger$ Defined in arbitrary but consistent units.

Table 1. Run specifications, isotropic cases.

$\begin{array}{lc}\text { Wavenumber shell } & \text { Number of points } \\ 1(2 \leqslant|\mathbf{k}|<4) & 26 \\ 2(4 \leqslant|\mathbf{k}|<6) & 66 \\ 3 & 158 \\ 4 & 234 \\ 5 & 410 \\ 6 & 470 \\ 7 & 738 \\ 8 & 866 \\ 9 & 1170 \\ 10 & 1358 \\ 12 & 1626 \\ 13 & 1970 \\ 14(28 \leqslant|\mathbf{k}| \leqslant 30) & 2366 \\ 15\left(30 \leqslant|\mathbf{k}|<k_{\max }\right) & 2538 \\ k_{\max }=2(242) \frac{1}{2} \approx 31 \cdot 2 & 1162 \\ & \text { TABLE } 2\end{array}$

A smaller value of $n$ is obtained if the relative energy content at very low wavenumbers is increased, since both the viscous dissipation and the nonlinear energy transfer are reduced at very low wavenumbers (Batchelor 1959, p. 155). Therefore an initial energy spectrum of the form

$$
\widehat{E}(k, 0)=v_{0}^{2} k_{\text {peak }}^{-2} k \exp \left[-k / k_{\text {peak }}\right]
$$

might be more appropriate. In this study we use both types of initial energy spectrum. As we are unable to extend the numerical experiments to higher Reynolds numbers, we include also some cases with rather low Reynolds numbers so that one may extrapolate to higher Reynolds numbers. The initial values for the several runs are given in table 1. Run $I 1$ is statistically identical to run 4 of $\mathrm{Kraichnan}$ (1964) (who used the direct-interaction approximation) and rin 3 of Orszag \& Patterson (1972b), except that they used $\Delta t=0.004$. The truncation errors caused by the larger time increment used here have been found to be negligible. Statistical results in wavenumber space are obtained by summing over all wavenumbers lying within a shell $k-\Delta k \leqslant|\mathbf{k}|<k+\Delta k$ $(\Delta k=1)$. The number of discrete wavenumber vectors falling in the first shells is small 


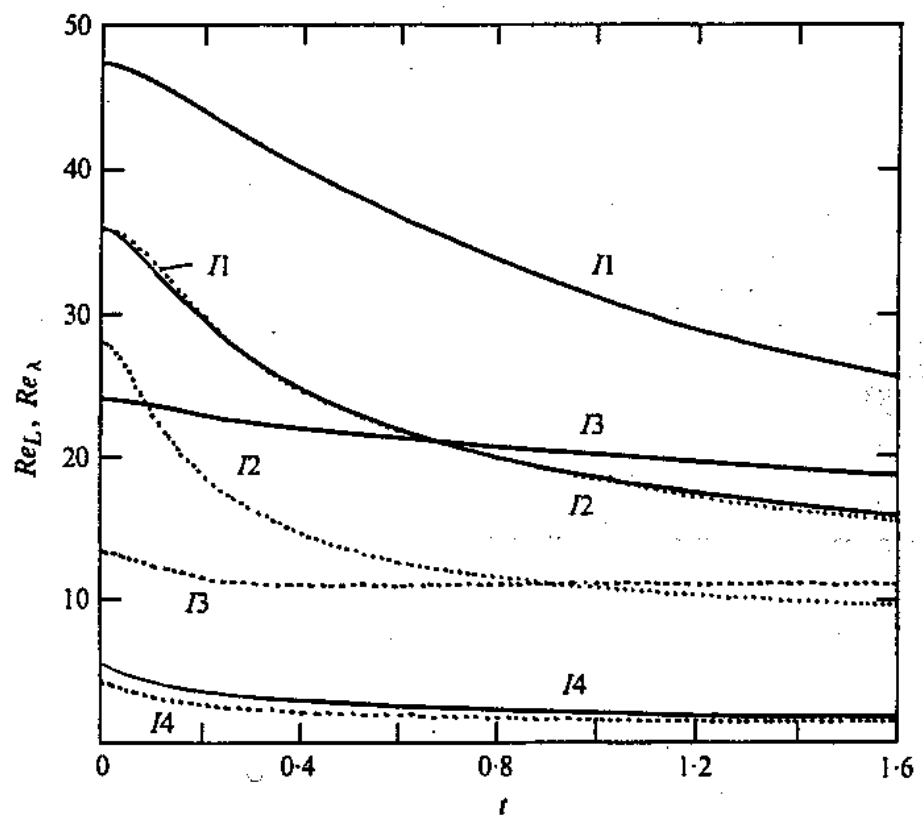

Figune 1. Reynolde numbers vs. time. - $R e_{L} ; \cdots \cdots, R e_{\lambda}$.

(see table 2). The statistical errors are not small for those quantities which are mainly dependent upon the low wavenumber range, and it is therefore desirable to shift the peak (at $k=k_{\text {peak }}$ ) of the energy spectrum to higher wavenumbers. This has been done for cases I2-I4. However, this increases the truncation errors at the high wavenumber cut-off and thus diminishes the maximum Reynolds number that can be simulated aceurately. Figure 1 shows the Reynolds numbers $R e_{L}$ and $R e_{\lambda}$ based on the integral scale $L_{f}$ and microscale $\lambda$, respectively, as a function of time $t$. The definitions of these quantities are

$$
\begin{aligned}
R e_{L} & =v L_{f} / \nu, \quad R e_{\lambda}=v \lambda / \nu, \\
L_{f}(t) & =\frac{\pi}{2[v(t)]^{2}} \int_{0}^{\infty} \frac{E(k, t)}{k} d k=\frac{1}{v^{2}} \int_{0}^{\infty} R_{l}^{l}(r) d r, \\
\lambda(t) & =\left(15 \nu[v(t)]^{2} / \epsilon(t)\right)^{\frac{1}{2}},=\left[\left\langle u_{1}^{2}\right\rangle /\left\langle\left(\partial u_{1} / \partial x_{1}\right)^{2}\right\rangle\right]^{\frac{1}{2}}, \\
V(t) & =\left\langle\frac{1}{3} \mathbf{u}(t) \cdot \mathbf{u}(t)\right\rangle^{\frac{1}{2}}, \\
\epsilon(t) & =2 v \int_{0}^{\infty} k^{2} E(k, t) d k=\nu\left\langle\left(\frac{\partial u_{i}}{\partial x_{k}} \frac{\partial u_{i}}{\partial x_{l k}}\right)\right\rangle, \\
R_{l}^{l}(r) & =\left\langle u_{1}(\mathbf{x}) u_{1}\left(\mathbf{x}+r \mathrm{e}_{1}\right)\right\rangle, \quad \mathrm{e}_{1}=(1,0,0) .
\end{aligned}
$$

Here $v$ is the root-mean square (r.m.s.) velocity, $\nu$ is the kinematic viscosity, $\epsilon$ is the viscous energy dissipation rate, $R_{l}^{l}$ is the longitudinal second-order two-point velocity correlation and the angular brackets denote averaging over the box. The effects of high wavenumber truncation can be seen from figure 2 , where the skewness

$$
S=-\left\langle\left(\partial u_{1} / \partial x_{1}\right)^{3}\right\rangle\left\langle\left(\partial u_{1} / \partial x_{1}\right)^{2}\right\rangle^{-\frac{3}{2}}
$$

is plotted $v s$. time for the four runs. The skewness measures the energy transport from low to high wavenumbers by the inertial terms. Any decrease in its value (e.g. as for run I3) indicates that this energy transport is disturbed by the high wavenumber cut-off, in that the energy transport is reflected at this cut-off and retransported to 


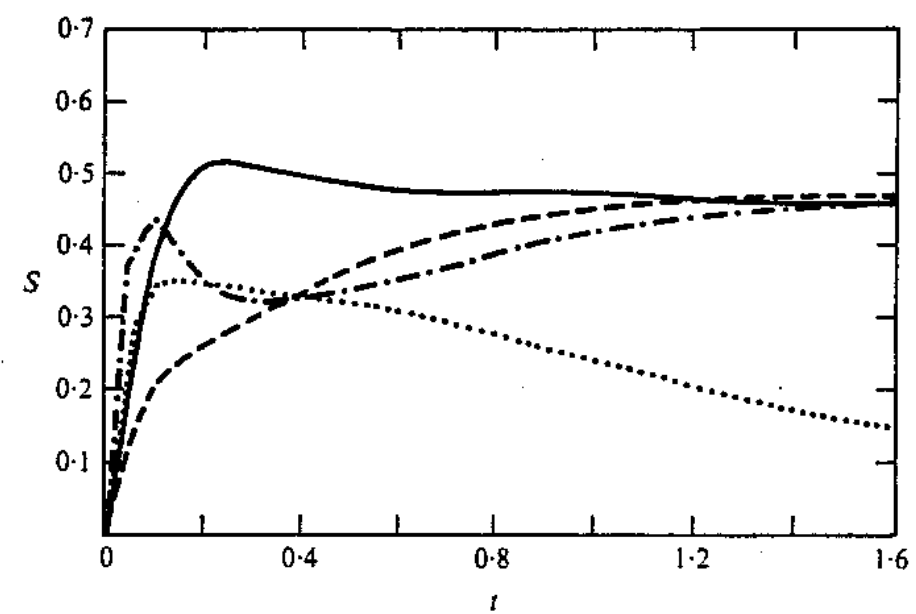

FIGORE 2. Skewness coefficient $v 8$. time. —, I1; - - I2; - -,$I 3 ; \cdots \cdots, I 4$.

lower wavenumbers. The reduction of $S$ for very low Reynolds numbers (case I4) corresponds to the reduction of nonlinear terms by the viscous damping and is in accordance with recent measurements by Tavoularis (1977), who found a reduction of $S$ for $R e_{\lambda}<5$.

From figure 1, we see for run $I 3$ that the Reynolds number $R e_{\lambda}$ becomes virtually independent of time, indicating the approach to a self-similar state. Such behaviour was predicted for the type of energy spectrum given by (10) by Kraichnan (1964), Leith (1967) and Herring (1973) using different turbulence models.

Figure 3 shows some properties of run $I 1$ at $t=0.2$ in an $x_{1}, x_{2}$ plane. The vector plot in figure $3(a)$ shows the velocity components $u_{1}$ and $u_{2}$. The vorticity component $\omega_{1}$, the kinetic energy $E$ and the pressure $p$ are given by contour plots. These plots give a general impression of the range of scales covered by the present method. The threedimensional space distribution is illustrated for the same run and time in figure 4, where perspective views of three-dimensional 'clouds' are plotted. In figure $4(a)$, within the clouds the kinetic energy $E$ is larger than $\frac{1}{2} E_{\max }(=3 \cdot 365)$; in figure $4(b)$ the fluctuating pressure (mean value zero) is smaller than $p_{s}=\frac{1}{3} p_{\operatorname{m1n}}(=-1.431)$. Some positive correlation between energy and negative pressure, which would be expected if Bernoulli's law holds for turbulent flow, can be recognized.

The time evolution of the spectral distribution is shown in figure $5(a)$ for case $I 4$. Perspective plots of the kinetic energy $\hat{E}(k, t)$, the dissipation $\hat{\varepsilon}(k, t)$, the pressure $\hat{p}(k, t)$ and the transfer spectrum $\hat{T}(k, t)$ as functions of the wavenumber $k(=K)$ and time $t(=T)$ are shown. The same can be seen for case $I 1$ in figure $5(b)$. The main difference between these runs can be seen from the dissipation plots. Whereas the dissipation rate clearly grows at high wavenumbers during the initial phase of run $I 1$ (owing to the inertial energy transport), no such piling up can be seen for run 14 . On the other hand, the energy is distributed over a large number of wavenumber shells in run 14 , so we can expect good accuracy for this run. For both cases the effect of energy loss at low wavenumbers and of energy gain at high wavenumbers by means of the inertial terms can be seen from the transfer spectra, which are defined as

$$
\hat{\Upsilon}\left(k_{0}\right)=\sum_{k_{0}-\Delta k \leqslant|k|<k_{0}+\Delta k} \hat{\mathbf{u}}(\mathbf{k}) \cdot \hat{\tau}(-k) .
$$




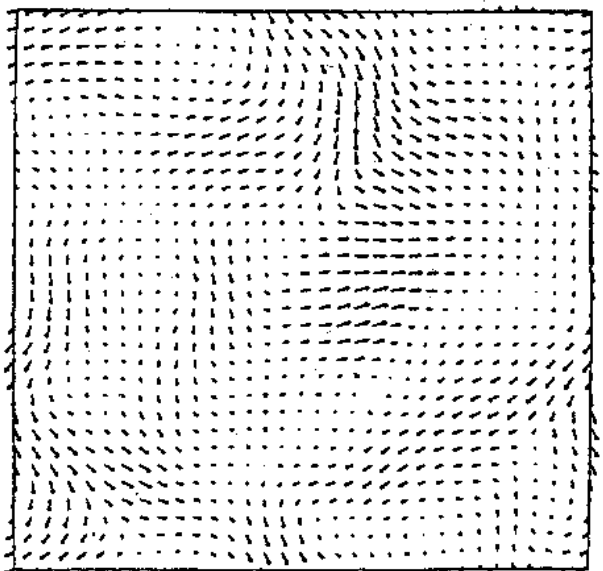

(a)

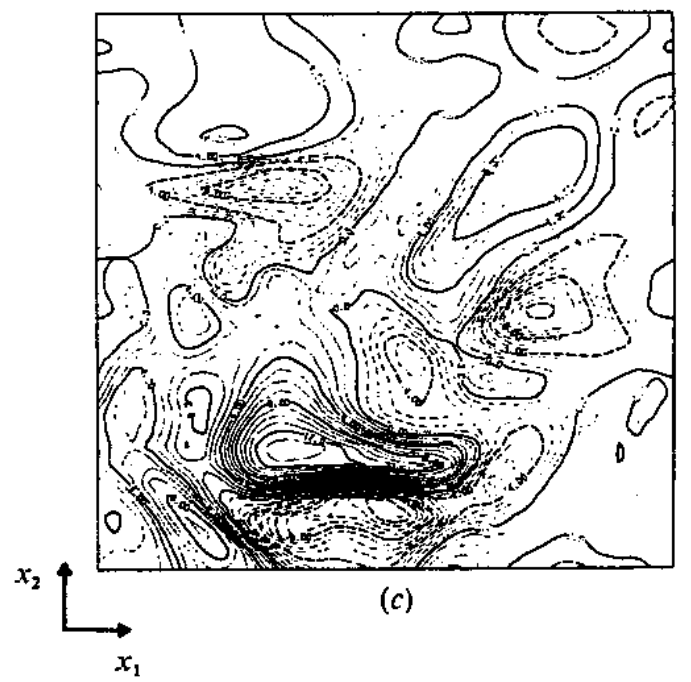

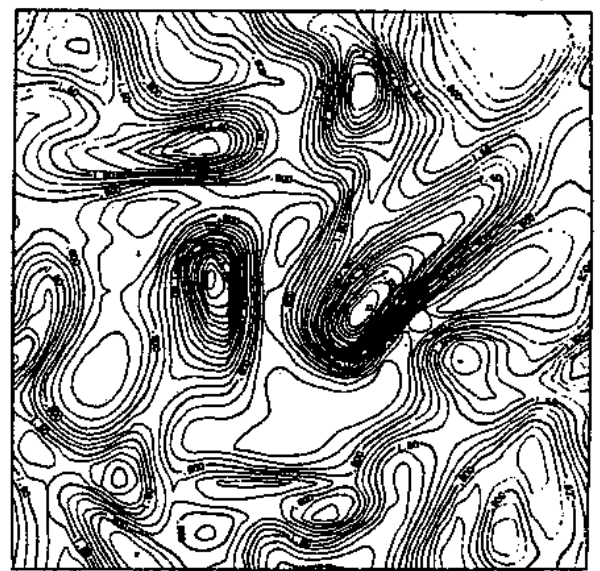

(b)

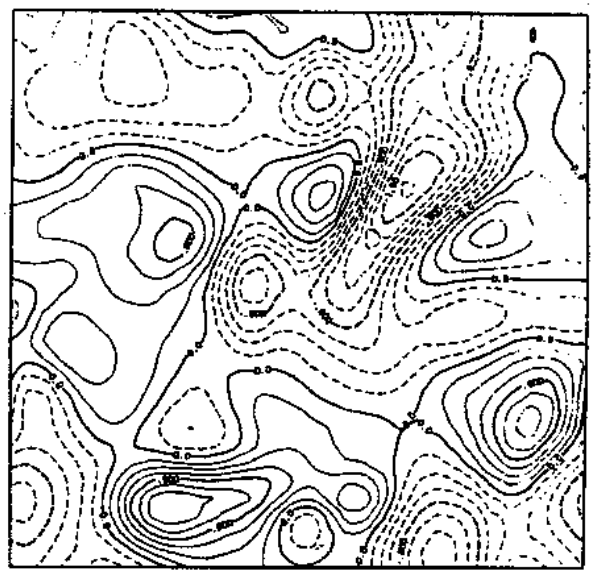

(d)

FIGURe 3. (a) Velocities $\left\{u_{1}, u_{2}\right\},(b)$ kinetic energy $E$, (c) vorticity $\omega_{1}$ and $(d)$ pressure $p$ for run $I 1$ at time $t=0.2$ in an $x_{1}, x_{2}$ plane. The maximum vector corresponds to a value of 2.71 for the magnitude of the velocities. The contour intervals are 0.2 for energy and pressure and 1.0 for the vorticity.

An inertial range, where $\hat{Y}(k) \approx 0$, does not exist for these Reynolds numbers. The little 'bumps' in the spectral distribution appear because the number of discrete wavenumber points in the shells is not strictly proportional to $k^{2}$, as can be seen from table 2. For runs $I 2$ and $I 3$ the above-mentioned truncation errors become obvious from the dissipation spectra shown in figure 6 and from the values of the ratio $k_{K} / k_{\max }$ plotted in figure 7 ; here $k_{K}$ is the Kolmogorov wavenumber $k_{K}=\left(\varepsilon / \nu^{3}\right)^{\frac{1}{t}}$ and $k_{\max }$ is the maximum of the resolved wavenumbers $\left(k_{\max }=31.2\right.$ in this simulation). Above the Kolmogorov wavenumber the energy content and the dissipation rate are sufficiently small to justify the truncation. However, we shall see that the main results discussed in the following chapters are nearly independent of both the initial conditions and time as they are mainly dependent upon the low wavenumber range. 


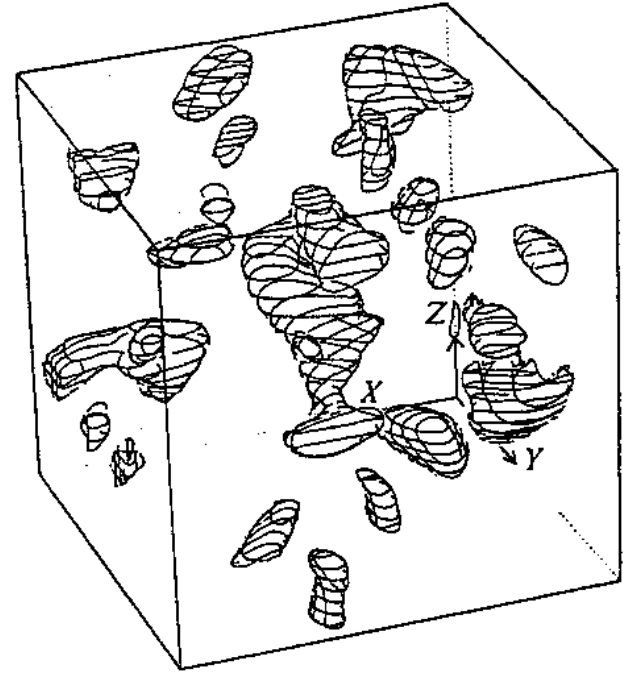

(a)

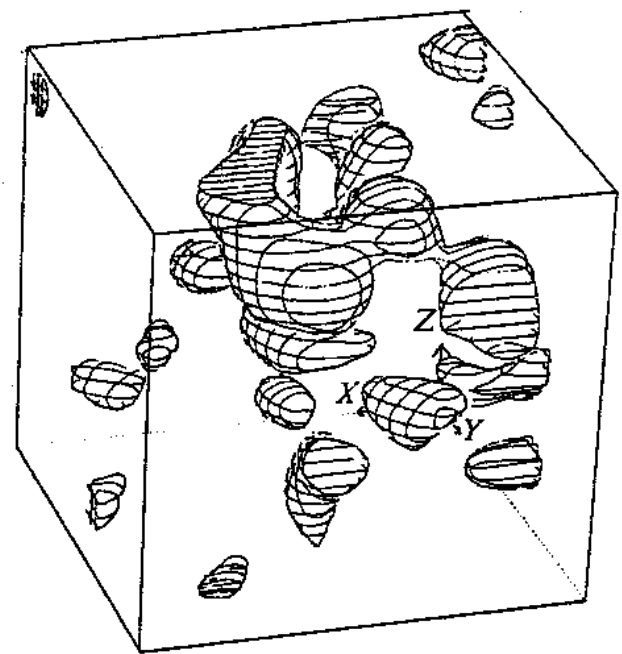

(b)

FigdRE 4. Isosurfaces of $(a)$ kinetic energy $E$ and $(b)$ pressure $p$ in real space $\left(x_{1}, x_{2}, x_{3}\right)=(X, Y, Z)$ for run $I 1$ at time $t=0.2$. Inside the 'clouds' $E \geqslant 3.365=\frac{1}{2} E_{\max }$ and $p \leqslant-1.431=\frac{1}{3} p_{\text {min }}$.

Some departure from isotropy is illustrated by figures $8(a)$ and $(b)$, which show the energy ratios

$$
r_{21}=\hat{E}_{22}(k, t) / \hat{E}_{11}(k, t), \quad r_{31}=\widehat{E}_{33}(k, t) / \widehat{E}_{11}(k, t),
$$

where $E_{i j}(k, t)$ is the energy tensor evaluated from

$$
\hat{E}_{i j}\left(k_{0}, t\right)=\sum_{k_{0}-\Delta k \leqslant|\mathbf{z}|<k_{0}+\Delta k} \hat{\mathbf{u}}_{i}(\mathbf{k}, t) \hat{\mathbf{u}}_{j}(-\mathbf{k}, t) .
$$

The ratios $r_{21}$ and $r_{31}$ should be equal to one everywhere in isotropic turbulence. We find, however, that in the final period of the decay these ratios tend to approach some asymptotic value different from one, especially at low wavenumbers. This behaviour seems to be a consequence of the small number of points and the resulting anisotropy in the first shell. This anisotropy becomes dominant after some time (which is shorter for lower Reynolds numbers) as the low wavenumber modes decay considerably more slowly than the high wavenumber modes. Similar results have been found by Batchelor \& Stewart (see Batchelor 1959, figure 5.3), Uberoi (1963) and Bennett (1976) for grid turbulence, where the lowest wavenumbers are anisotropic owing to the grid. In our case, however, this effect should disappear if we average over large ensembles.

\subsection{Pressure and fourth-order velocity statistics}

The pressure field is completely determined when the velocity field is known. Therefore theories have been developed which predict the correlations between pressure fluctuations and any other quantity for isotropic turbulence (Batchelor 1951, 1959, \$8.3; Uberoi 1953, 1954; Limber 1951; Kraichnan 1956a). In these theories fourthorder velocity correlations appear which are not known a priori. All theories have assumed that the quadruple correlations are related to the double correlations by the following:

$$
\left\langle u_{i} u_{j} u_{k} u_{l}\right\rangle=\left\langle u_{i} u_{k}\right\rangle\left\langle u_{j} u_{l}\right\rangle+\left\langle u_{i} u_{l}\right\rangle\left\langle u_{j} u_{k}\right\rangle+\left\langle u_{i} u_{j}\right\rangle\left\langle u_{k} u_{l}\right\rangle
$$



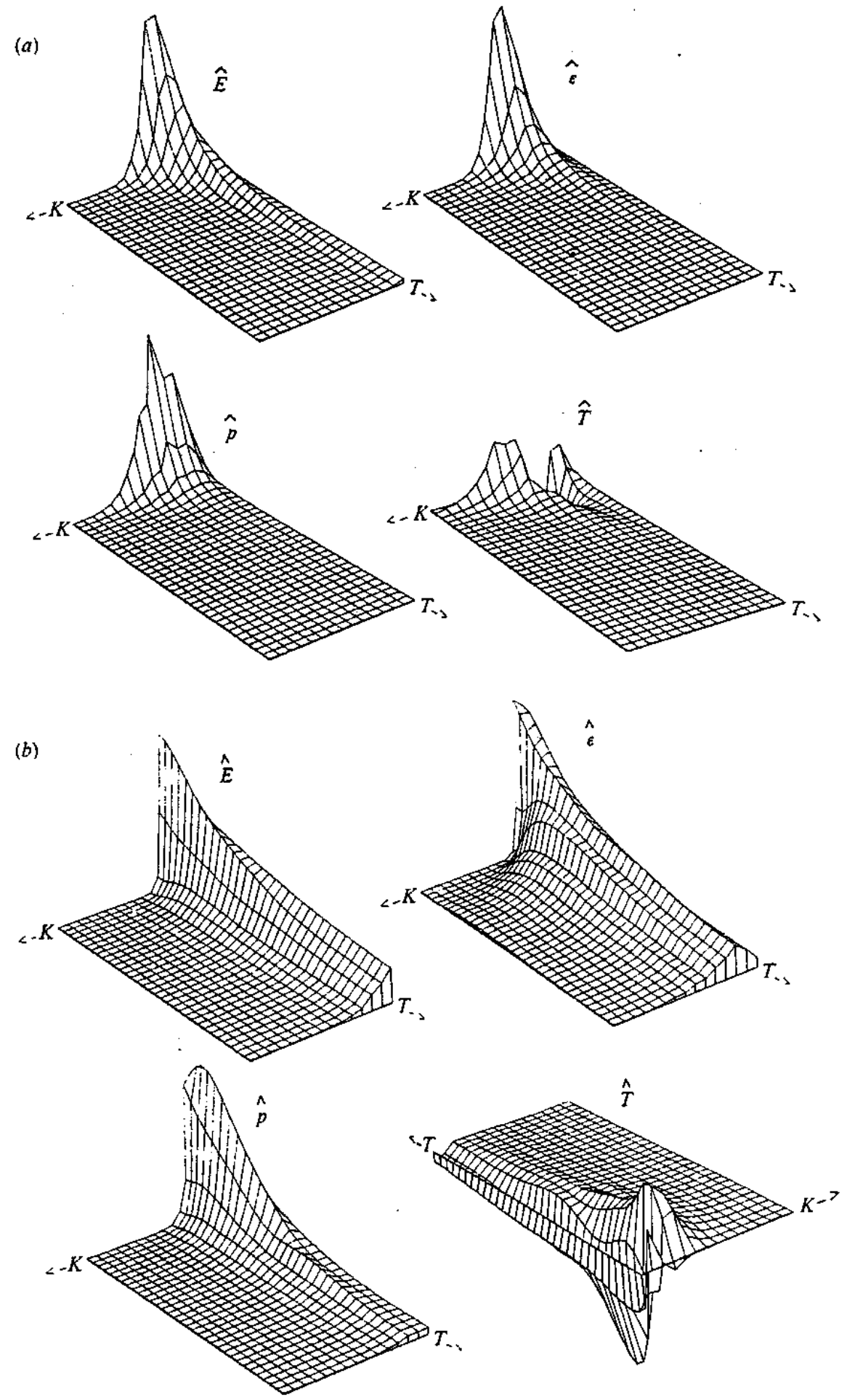

FIGURE 5. Spectral distribution of the kinetic energy $\hat{E}$, pressure $\hat{p}$, dissipation $\epsilon$ and transfer rate $\hat{\Upsilon} v s$. wavenumber $(k=K, 3 \leqslant k \leqslant 31)$ and time $(t=T, 0 \leqslant t \leqslant 1 \cdot 6)$ for $(a)$ run $I 4$ and (b) run $I 1$. 


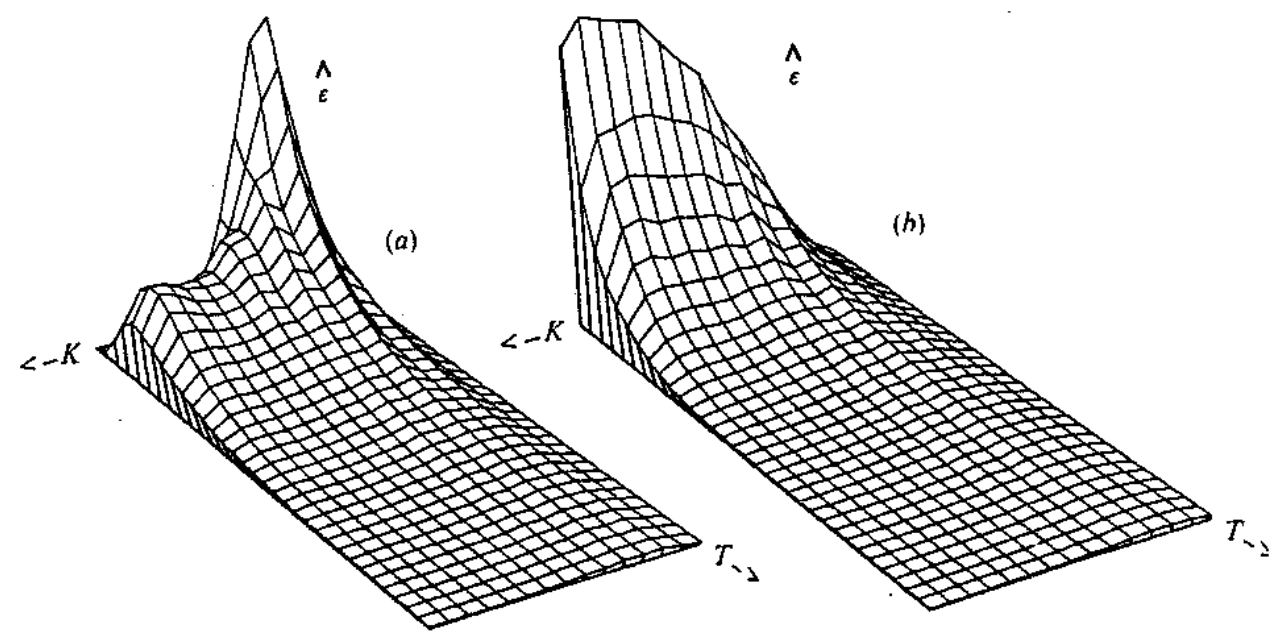

Frgute 6. Spectral distribution of dissipation $\hat{\epsilon}(k, t)$ for $(a)$ run $I 2$ and (b) $I 3$.

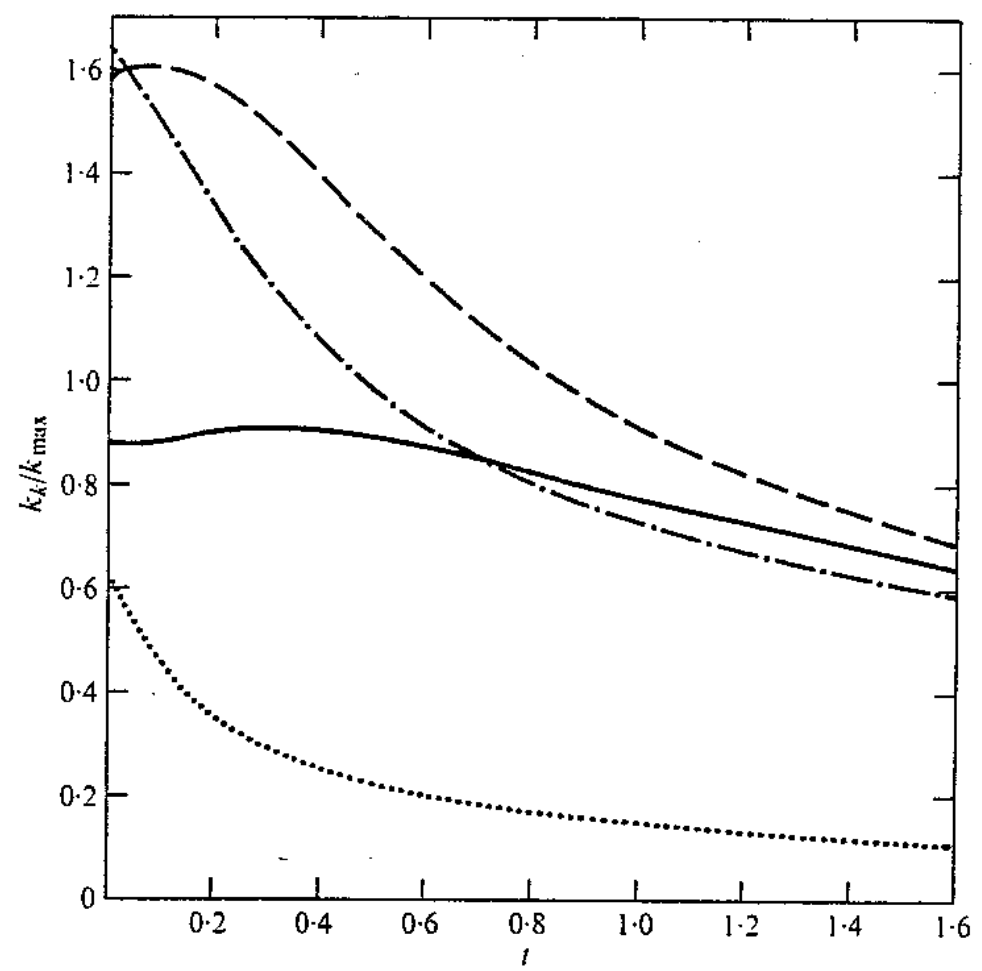

FIGURE 7. Kolmogorov wavenumber $k_{K}$ normalized by the maximum resolved wavenumber $k_{\max }$ vs. time. -,$I 1 ;-\cdots, I 2 ;-.-, I 3 ; \cdots \cdots, I 4$.

Here the velocities are not necessarily defined at the same point. This hypothesis is true if the velocity distribution is Gaussian. But even without this condition (which is not true for turbulence, since the triple correlations are not all zero) the above assumption might be valid.

This assumption has been supported for the velocity field by several experiments (e.g. Uberoi 1953; Batchelor 1959, § 8.1; Frenkiel \& Klebanoff 1967; Kuo \& Corrsin 1971). The later experiments do show, however, large departures from normality for 

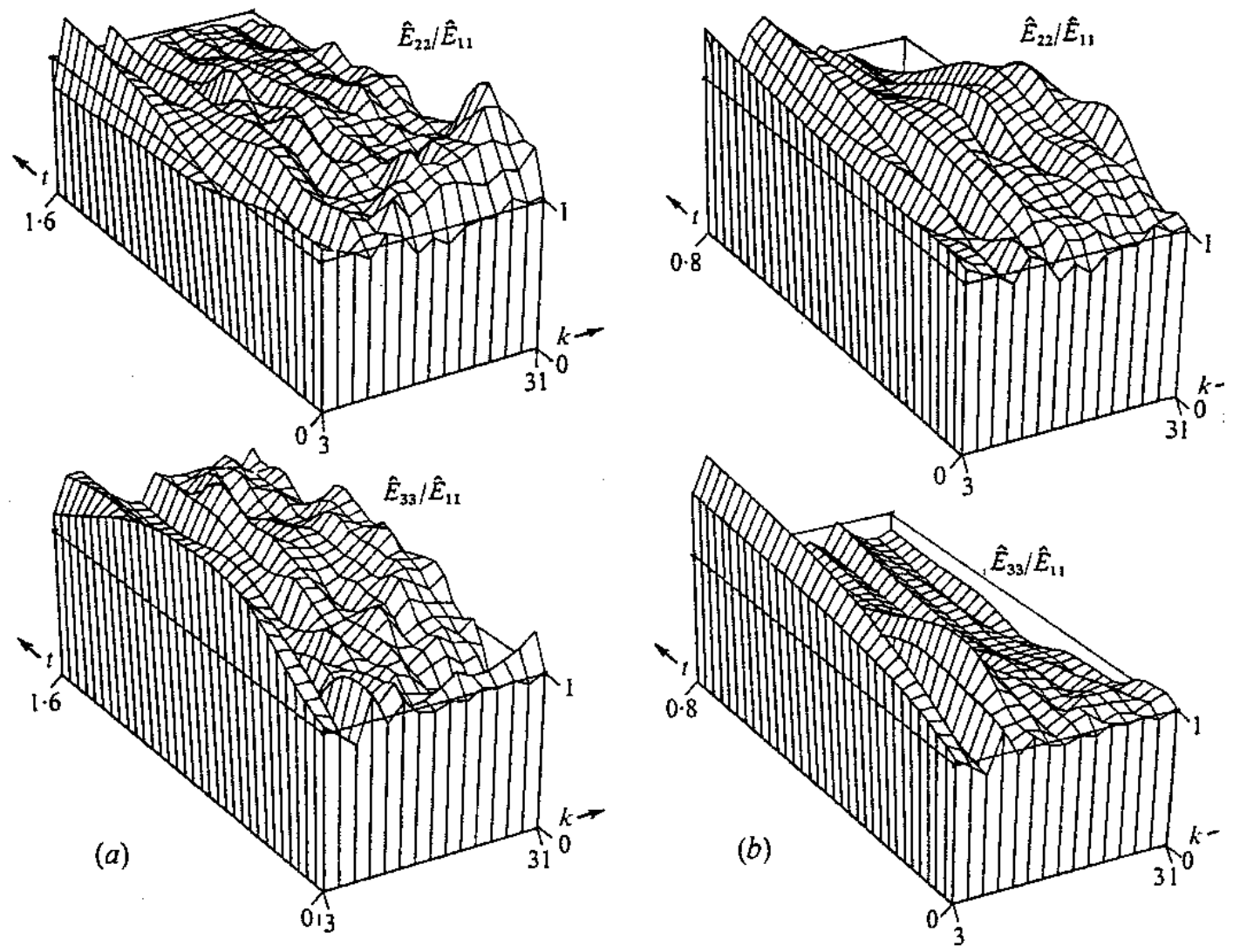

FIGURE 8. Energy ratios vs. wavenumber and time for $(a)$ run $I 1$ and $(b)$ run $I 4$.

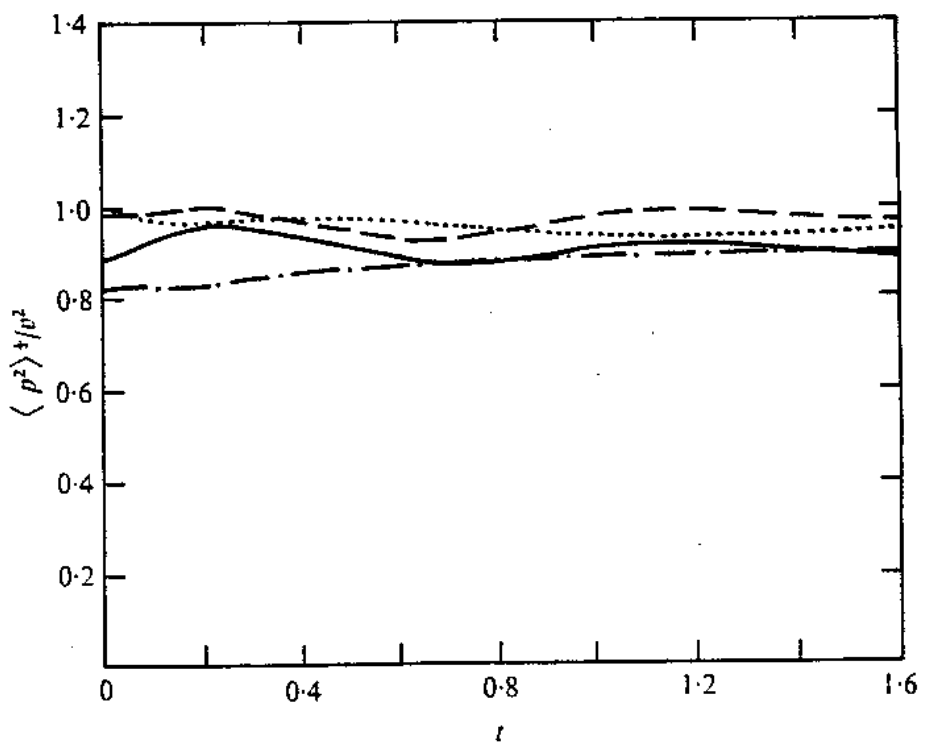

Figure 9. R.m.s. pressure fluctuation normalized by mean-square velocity fluctuations vs. time.,$- I 1 ;---, I 2 ;-\cdot-, I 3 ; \cdots \cdots, I 4$. 


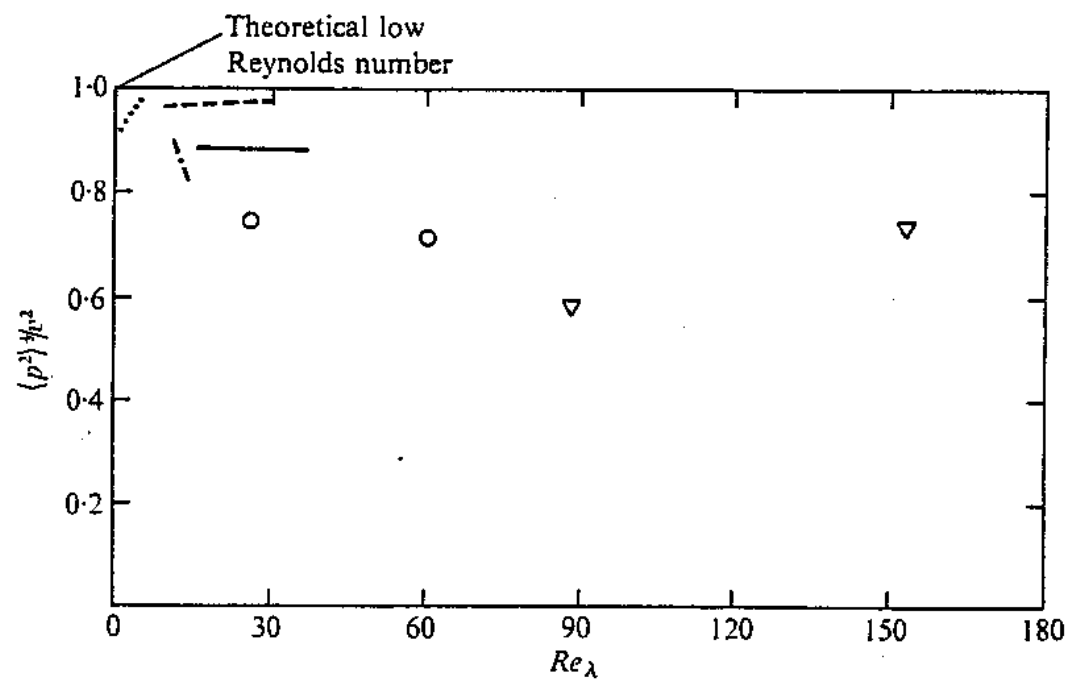

FigURE 10. R.m.s. pressure fluctuations normalized by mean-Bquare velocity fluctuations vs. Reynolds number. Theory: -- , Uberoi (1953), high Reynolds numbers. Experiments: $O$, Uberoi (1953); $\nabla$, Liepmann et al. (see Uberoi 1954). Present results: $\longrightarrow, I 1 ;---, I 2 ;-.-$ $I 3 ; \cdots \cdots, I 4$.

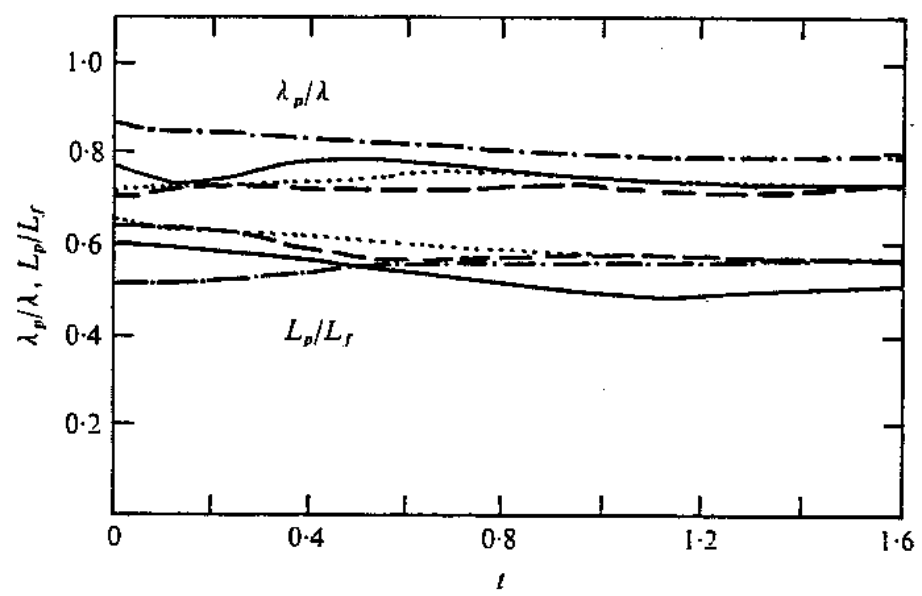

FIGURE 11. Ratio of microscales $\left(\lambda_{p} / \lambda\right)$ and integral scales $\left(L_{p} / L_{f}\right)$ for pressure and velocity $v s$. time. -,$I 1 ;---, I 2 ;-\cdot-, I_{3}^{3} ; \cdots \cdots, I 4$.

the time derivatives of the velocity (which is consistent with the numerical results for the skewness plotted in figure 2).

At very low Reynolds numbers $\left(R e_{\lambda} \leqslant 15\right.$, Batchelor \& Townsend 1948) the longitudinal double velocity correlation $R_{l}^{l}(r)$ (defined in $\S 3.1$ ) is expected to be equal to the self-similar form it approaches under pure viscous decay:

$$
R_{l}^{l}(r)=v^{2}\left[\exp -r^{2} /\left(2 \lambda^{2}\right)\right]
$$

This assumption allows one to evaluate the pressure statistics quantitatively and will also be checked.

Figure 9 shows the computed r.m.s. value of the pressure fluctuations normalized by the actual mean-square value of the velocity fluctuations as a function of time for 


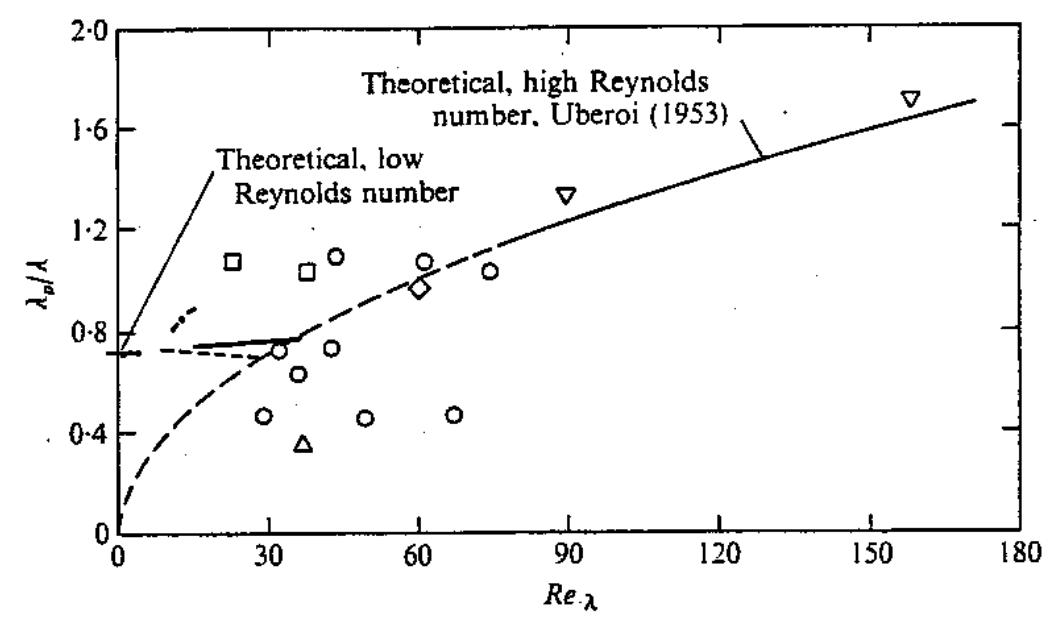

FIGURE 12. Ratio $\lambda_{p} / \lambda$ of microscales for pressure and velocity fluctuations vs. Reynolds number. Experiment: $\triangle$, Simmons (1935); $\square$, Collis (1948); $O$, Uberoi \& Corrsin (1953); $\diamond$, Uberoi (1953); $\nabla$, Liepmenn et al. (see Uberoi 1954). Present results:,$- I 1 ;---, I 2 ;-.-, I 3$; $\cdots, \ldots, I 4$.

the different runs. We find that this ratio is nearly independent of the initial values and of time and is thus independent of the Reynolds number within the range considered here. The results are compared in figure 10 with those obtained experimentally by Uberoi (1953) and others cited therein. The experimental results are derived from second-order velocity correlations using (11). The theoretical value of unity for $R e_{\lambda}=0$ is based on (12). The numerical experiments give somewhat higher values, however, than do the laboratory experiments.

Next we report the resultant length scales $\lambda_{p}$ and $L_{p}$ as defined by Batchelor (1951). Figure 11 shows the ratio of the microscales $\lambda_{p}$ and $\lambda$, where $\lambda_{p}$ is a measure of the pressure gradient:

$$
\lambda_{p}=\left\langle p^{2}\right\rangle /\left\langle\left(\frac{\partial p}{\partial x_{1}}\right)^{2}\right\rangle
$$

From figure 11 we see that the ratio $\lambda_{p} / \lambda$ is again nearly independent of time and initial values. Figure 12 compares these results with those predicted theoretically and found experimentally from diffusion experiments (Batchelor 1951; Uberoi 1953). The theoretical value of $\left(\frac{1}{2}\right)^{\frac{1}{2}}$ for $\lambda_{p} / \lambda$ at $R e_{\lambda}=0$ is clearly confirmed. Moreover, the scatter of the numerical results appears to be smaller than that of the experimental results.

The large-scale spectrum of the pressure fluctuations is characterized by the integral length scale $L_{p}$ :

$$
\begin{gathered}
L_{p}=\frac{\pi}{2} \frac{1}{\left\langle p^{2}\right\rangle} \int_{0}^{\infty} \hat{p}(\mathbf{k}) \cdot \hat{p}(-\mathbf{k}) /|\mathbf{k}| d \mathbf{k}=\frac{1}{\left\langle p^{2}\right\rangle} \int_{0}^{\infty} R_{p}^{p}(r) d r, \\
R_{p}^{p}(r)=\left\langle p(\mathbf{x}) p\left(\mathbf{x}+r \mathrm{e}_{1}\right)\right\rangle .
\end{gathered}
$$

The ratio $L_{p} / L_{f}$ is also plotted $v s$. time in figure 11 . Kraichnan (1956a) has proved that $L_{p} / L_{f}<1$. Batchelor estimated $L_{p} / L_{f} \approx 0.54$. From the correlation functions given by Uberoi $(1953,1954)$ we estimate that

$$
L_{f} \approx 0.71 \text { in., } \quad L_{p} \approx\left\{\begin{array}{l}
0.57 \mathrm{in} . \\
0.2 \div 0.7 \mathrm{in} .
\end{array}\right.
$$



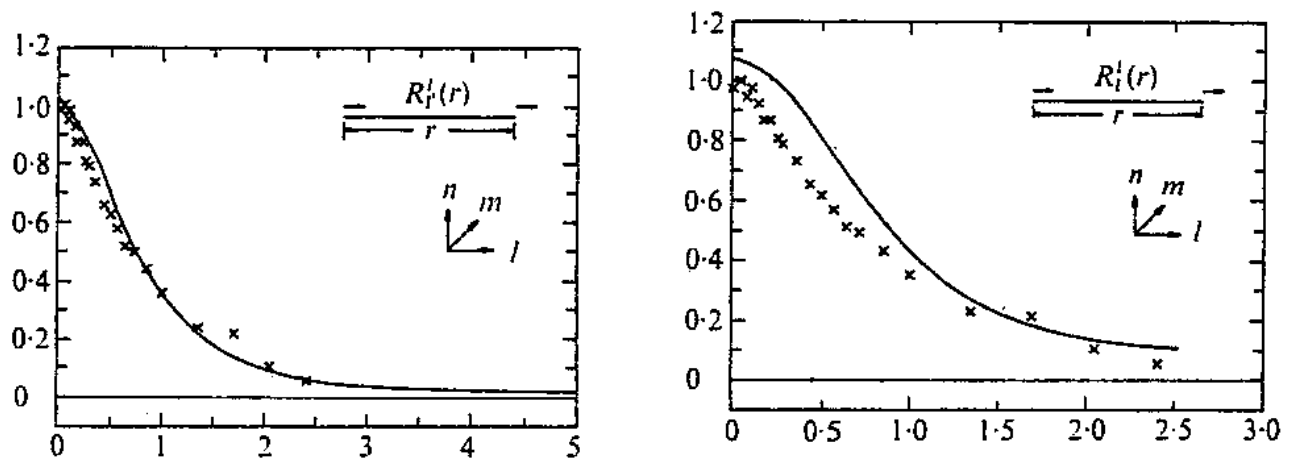

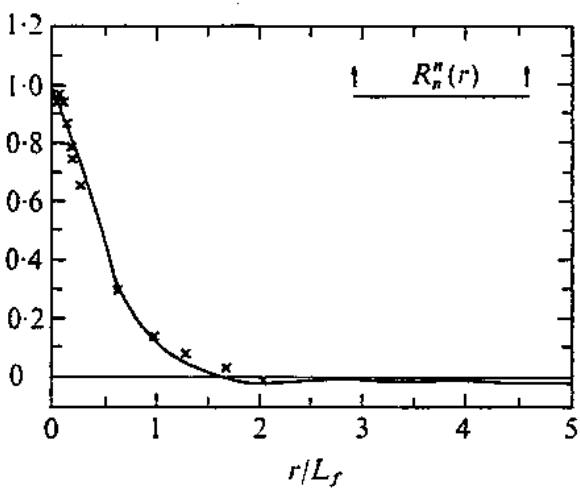

(a)

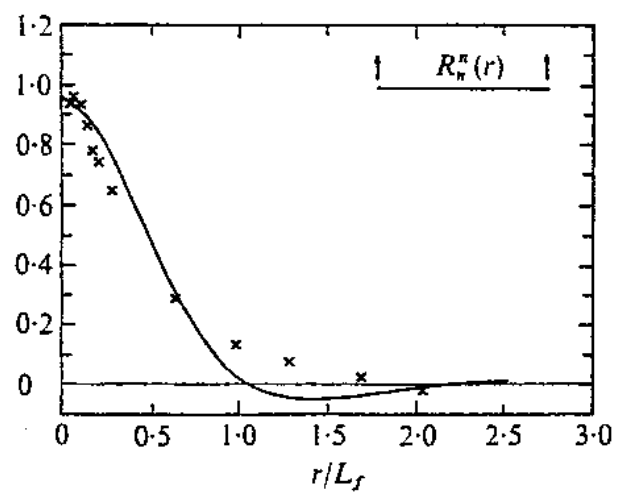

(b)

FraURe 13. Two-point second-order velocity correlations normalized by mean-square velocity fluctuations $v^{2}$ at time $t=0.2 v s$. normalized distance for $(a)$ run $I 3\left(L_{f}=0.32\right.$ ) and $(b)$ run $I 1$ $\left(L_{f}=0.62\right)$. The crosses represent the experimentel regults of Uberoi $(1953)\left(L_{f}=0.71 \mathrm{in}\right.$.).

The first value follows from the pressure correlation obtained from (11); the second pair of values follows from the results obtained directly from the measured fourth-order velocity correlations. Thus Uberoi's experiments give a value of $L_{p} / L_{f}$ between 0.3 and 1 , with a best estimate of 0.8 . The best estimate from the numerical simulations is $L_{p} / L_{f} \approx 0.55$ for the present range of Reynolds numbers.

The ratios $\left\langle p^{2}\right\rangle / v^{2}, \lambda_{p} / \lambda$ and $L_{p} / L$ certainly depend on the shape of the energy spectrum and thus depend on the initial values used in these simulations. In fact, such differences can be seen for $t=0$ in figures 9 and 11. These differences, although partly statistical errors, become smaller as time increases. This suggests that the importance of the different initial values decreases with increasing time.

In order to test the assumption of normality [equation (11)] we compute representatives of the fourth-order correlations

$$
\left\langle u_{i}(\mathbf{x}) u_{j}(\mathbf{x}) u_{k}(\mathbf{x}+\mathbf{r}) u_{l}(\mathbf{x}+\mathbf{r})\right\rangle, \quad\left\langle u_{i}(\mathbf{x}) u_{j}(\mathbf{x}) u_{k}(\mathbf{x}) u_{l}(\mathbf{x}+\mathbf{r})\right\rangle
$$

Using conditions of isotropy and continuity, the above correlations can be represented by four independent scalar functions (Uberoi 1953, 1954). These correlations are computed in real space using the $x_{3}$ direction as the direction of $\mathbf{r}$ and assuming invariance under rotation around this axis. Second-order correlation functions are also computed for comparison and are normalized by $v^{2}$. Fourth-order velocity correlations are normalized by $\left\langle u^{4}\right\rangle \equiv\left\langle\frac{1}{3}\left(u_{1}^{4}+u_{2}^{4}+u_{3}^{4}\right)\right\rangle$. Second-order pressure correlations and correlations between pressure and second-order velocity products are normalized by $v^{4}$. To make comparison possible, the separation length $r$ is normalized by the corre- 
sponding integral length scale $L_{f}(t)$. We show the results for $t=0.2$, a time that is large enough to allow triple correlations to develop and yet small enough to keep the errors due to departure from isotropy small.

The notation for these correlations, e.g.

$$
R_{n}^{n n}(|\mathbf{r}|)=\left\langle u_{l}(\mathbf{x}) u_{l}(\mathbf{x}) u_{n}(\mathbf{x}+\mathbf{r}) u_{n}(\mathbf{x}+\mathbf{r})\right\rangle /\left\langle u^{4}\right\rangle,
$$

is that of Uberoi (1953). The subscripts belong to those quantities (velocity component or pressure) which are defined at position $\mathbf{x}$ whereas the superscripts refer to the quantities at $\mathbf{x}+\mathbf{r}$. The values of these indices are $l, m, n$ or $p$, where $l$ refers to the velocity component parallel to $r, m$ to the velocity component in one possible direction perpendicular to $\mathrm{r}$ and $n$ to the third orthogonal component; $p$ is used if the quantity is the fluctuating pressure.

Figures $13(a)$ and $(b)$ show the resultant second-order correlations for runs $I 3$ and $I 1$, both at $t=0 \cdot 2$. We see reasonable agreement between measurements and the computations for run I3. Some departure from the isotropic state can be seen here in that the correlations are different from unity for $r=0$. (The general shape agrees even better for later times, but then the errors due to departure from isotropy increase.) Figures $14(a)$ and $(b)$ show the fourth-order velocity correlations for runs $I 3$ and $I 1$, for $t=0 \cdot 2$. Agreement between the computed values (full line) and the measured values is fair. Excellent agreement is found between the values computed directly and those which follow from the second-order correlations with use of (11). Figures 15(a) and $(b)$ show the pressure correlations. The second-order pressure correlations $R_{p}^{p}(r)$ are compared with the experimental results of Uberoi (1953), which have been computed from the measured second-order correlations using

$$
R_{p}^{p}(r)=2 \int_{r}^{\infty}\left(y-r^{2} / y\right)\left[\frac{d}{d y} R_{l}^{l}(y)\right]^{2} d y
$$

The dashed curve is the result obtained from (13) using the computed second-order velocity correlations. The latter agrees much better with the directly computed values than do the experimental values. It appears that the main errors in the experimental results come from the computation of the pressure statistics from measured velocity correlations. Uberoi $(1953,1954)$ himself remarks that because of experimental scatter and the need for computing differences and differentials the resultant pressure statistics are quite uncertain.

If $R_{l}^{l}(r)$ is given by (12), then (13) results in

$$
R_{p}^{p}(r)=\left(R_{l}^{l}(r)\right)^{2}
$$

This approximation is also plotted in figure 15 (dotted curve). We see that this approximation is not valid for run I3. It is valid, however, for run I4, where the Reynolds number is very low (see figure $15 b$ ). Some of the departure is due to anisotropy.

Figure 15 includes the results for the correlations between pressure and velocity products: $R_{i l}^{p}$ and $R_{n n}^{p}$. No experimental results are available for these correlation functions. Starting from the theory deduced by Limber (1951) [using (11)] we get

$$
\begin{aligned}
R_{l l}^{p}(r) & =\int_{0}^{r} \frac{7}{5} \frac{y^{4}}{r^{3}}\left(\frac{d}{d y} R_{l}^{l}\right)^{2} d y+\int_{r}^{\infty}\left[\frac{4}{5} \frac{r^{2}}{y}-\frac{1}{3} y\right]\left(\frac{d}{d y} R_{l}^{l}\right)^{2} d y \\
R_{n n}^{p}(r) & =-\int_{0}^{r} \frac{7}{10} \frac{y^{4}}{r^{3}}\left(\frac{d}{d y} R_{l}^{l}\right)^{2} d y-\int_{r}^{\infty}\left[\frac{1}{3} y+\frac{2}{5} \frac{r^{2}}{y}\right]\left(\frac{d}{d y} R_{l}^{l}\right)^{2} d y .
\end{aligned}
$$

(We take this opportunity to correct some errors in Uberoi (1954) for these correlations.) 

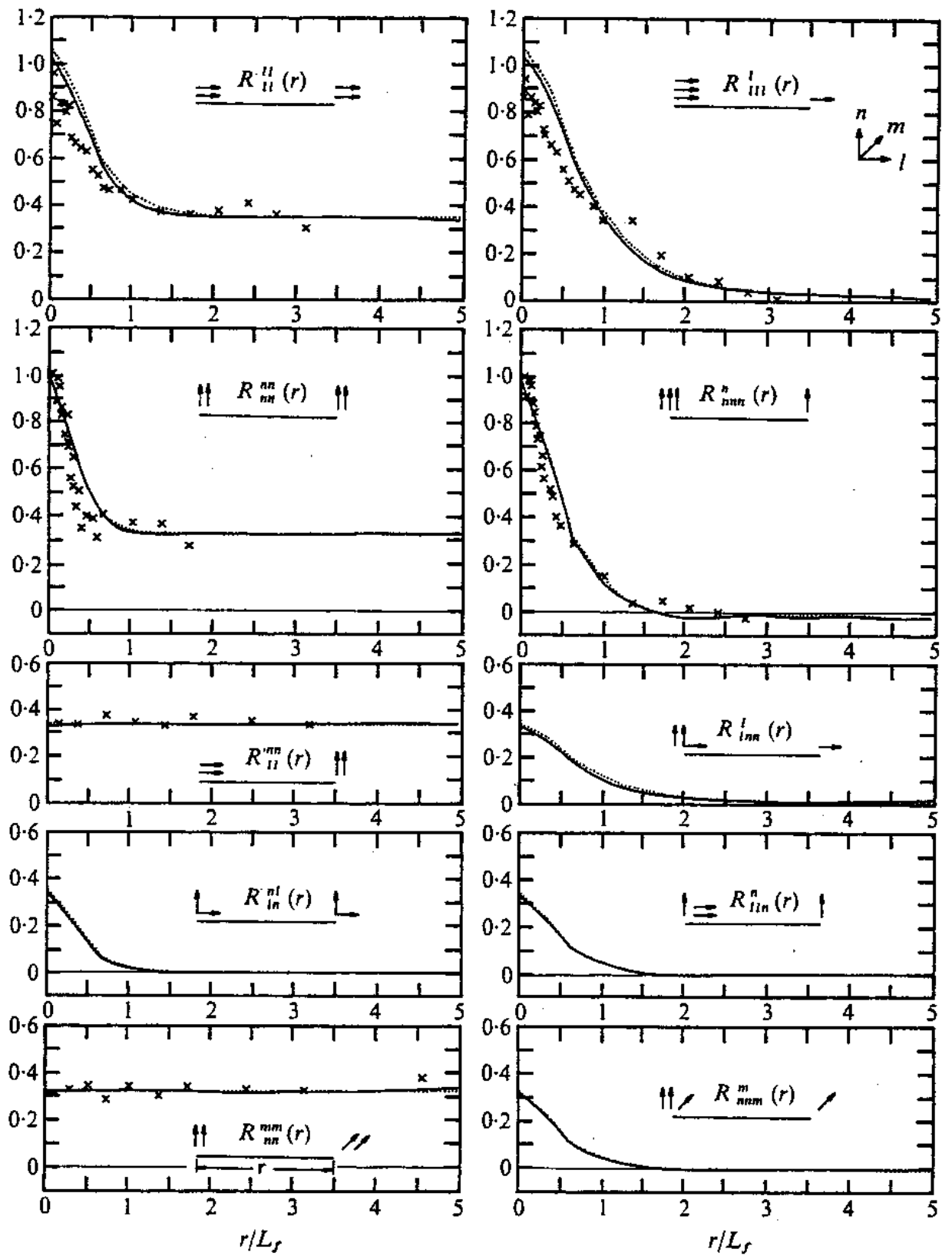

Figure 14(a). For legend see opposite.

If we use these results and (12) we get

$$
\begin{gathered}
R_{u l}^{p}(r)=v^{2}\left\{-\left[\frac{21}{8}\left(\frac{\lambda}{r}\right)^{2}+\frac{23}{12}+\frac{7}{15}\left(\frac{r}{\lambda}\right)^{2}\right] \exp \left[-(r / \lambda)^{2}\right]+\frac{21}{16} \pi^{\frac{1}{2}} \frac{\Phi(r / \lambda)}{(r / \lambda)^{3}}\right\}, \\
R_{n n}^{p}(r)=v^{2}\left\{\left[\frac{21}{16}\left(\frac{\lambda}{r}\right)^{2}+\frac{17}{24}-\frac{1}{60}\left(\frac{r}{\lambda}\right)^{2}\right] \exp \left[-(r / \lambda)^{2}\right]-\frac{21}{3} \frac{1}{2} \pi^{\frac{1}{2}} \frac{\Phi(r / \lambda)}{(r / \lambda)^{3}}\right\} \\
\Phi(x)=\frac{2}{\pi^{\frac{1}{2}}} \int_{0}^{x} \exp \left(-t^{2}\right) d t
\end{gathered}
$$

where 
Pressure and velocity fluctuations in turbulence
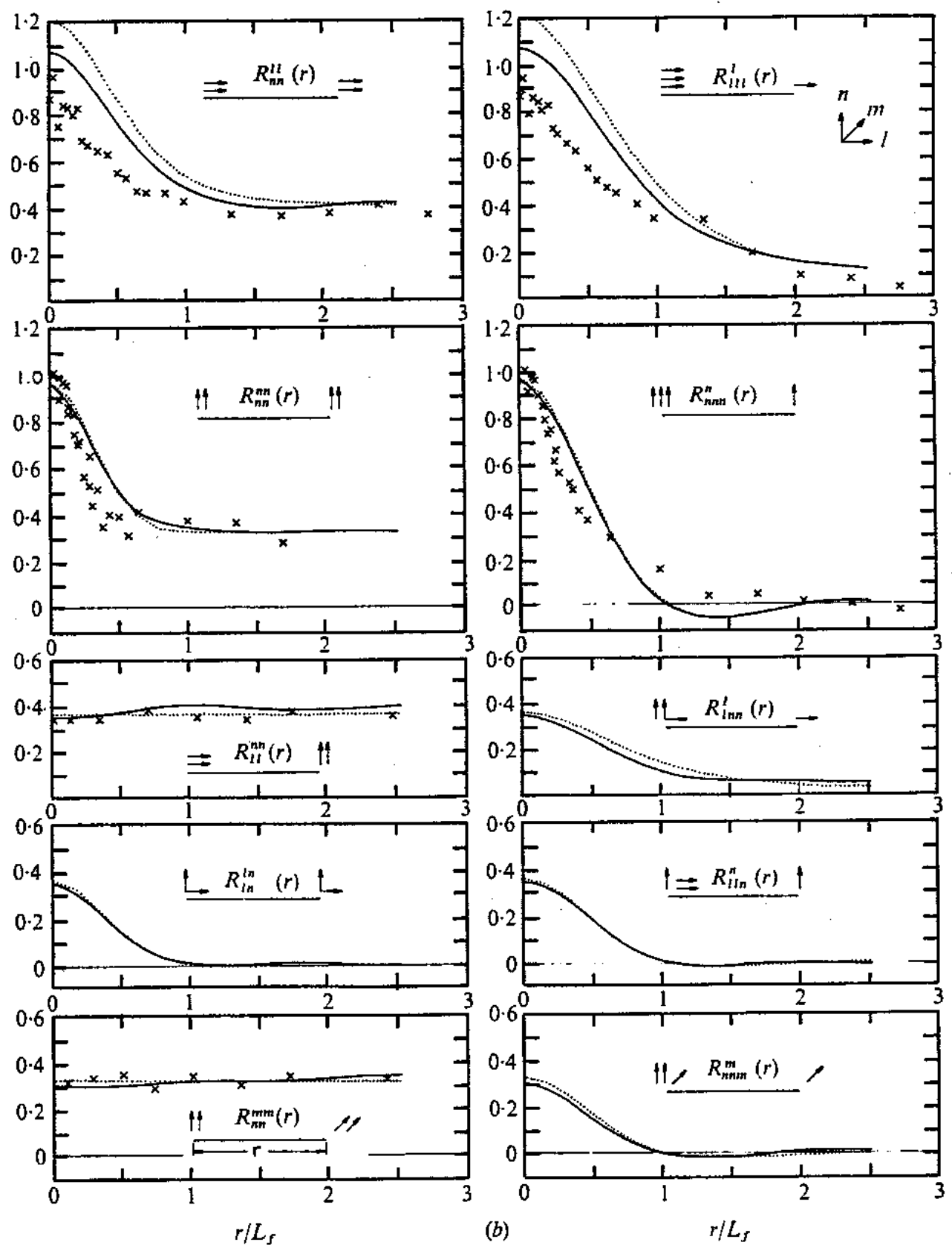

FigURE 14. Fourth-order two-point velocity correlations normalized by $1\left\langle u_{1}^{4}+u_{2}^{4}+u_{a}^{4}\right\rangle$ at time $t=0.2 v s$. normalized distance for $(a)$ run $I 3$ and $(b)$ run $I 1$. The crosses represent the experimental results of Uberoi (1953). The dotted curves are the predictions from second-order correlations assuming normality.

is the 'error integral'. For the given microscale $\lambda$, the above results were computed and are shown in figure $15(b)$. We see for run $I 4$ that the general shape is the same but that the amplitude of the variations is different. This might indicate some errors in the assumption [equation (11)]; however, the significance is small.

If (11) holds, the ratio $-2\left(R_{u l}^{p}(0)+2 R_{n n}^{p}(0)\right) / R_{p}^{p}(0)$ should be one (Uberoi 1954), the flatness factor $\left\langle\frac{1}{3}\left(u_{0}^{4}+u_{2}^{4}+u_{3}^{4}\right)\right\rangle / v^{4}$ should be three and the skewness $\left\langle\frac{1}{3}\left(u_{1}^{3}+u_{2}^{3}+u_{3}^{3}\right)\right\rangle / v^{3}$ 


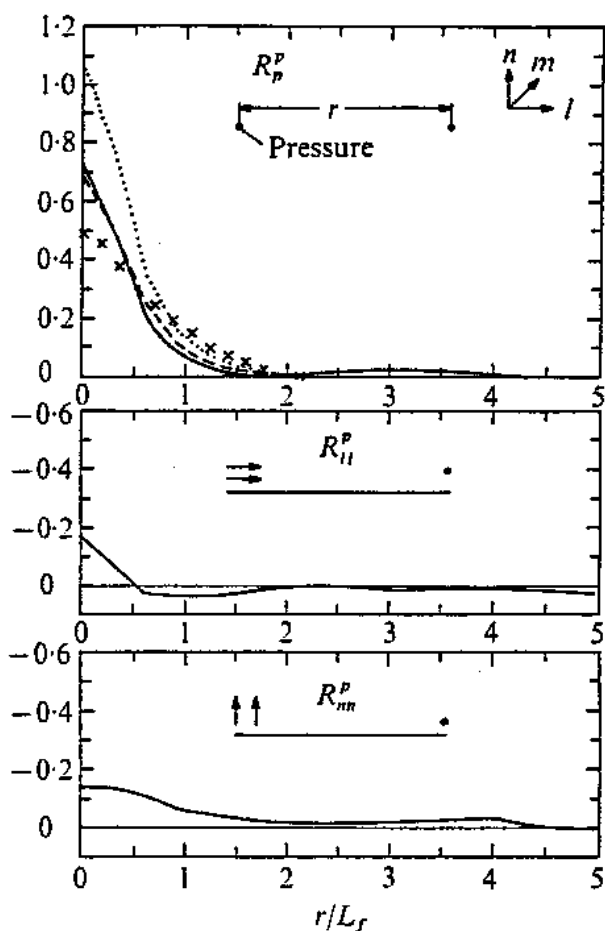

(a)

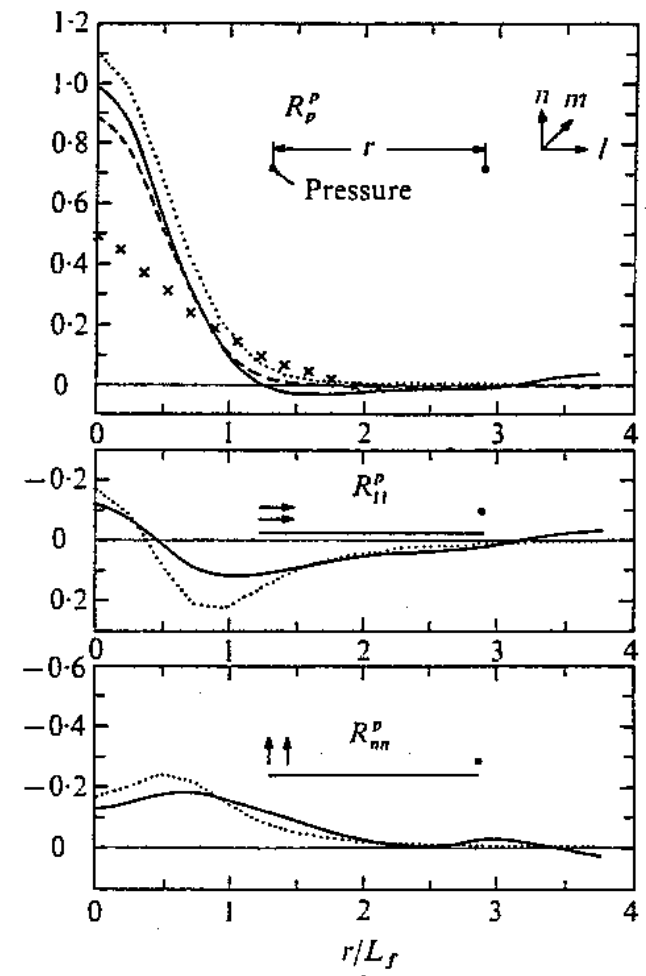

(b)

FIGURE 15. Pressure-velocity correlationg normalized by $v^{4}$ at time $t=0.2$ vs. normalized distance for (a) run $I 3$ and $(b)$ run $I 4$. The crosses represent the experimental results of Uberoi (1953). The dashed curve is the prediction from second-order correlations assuming normality. The dotted curve represents the assumption that (12) is also valid. (b) also shows the theoreticel behaviour of $R_{n n}^{p}$ and $R_{l l}^{p}$ (dotted curves) $(\lambda=0 \cdot 22)$.

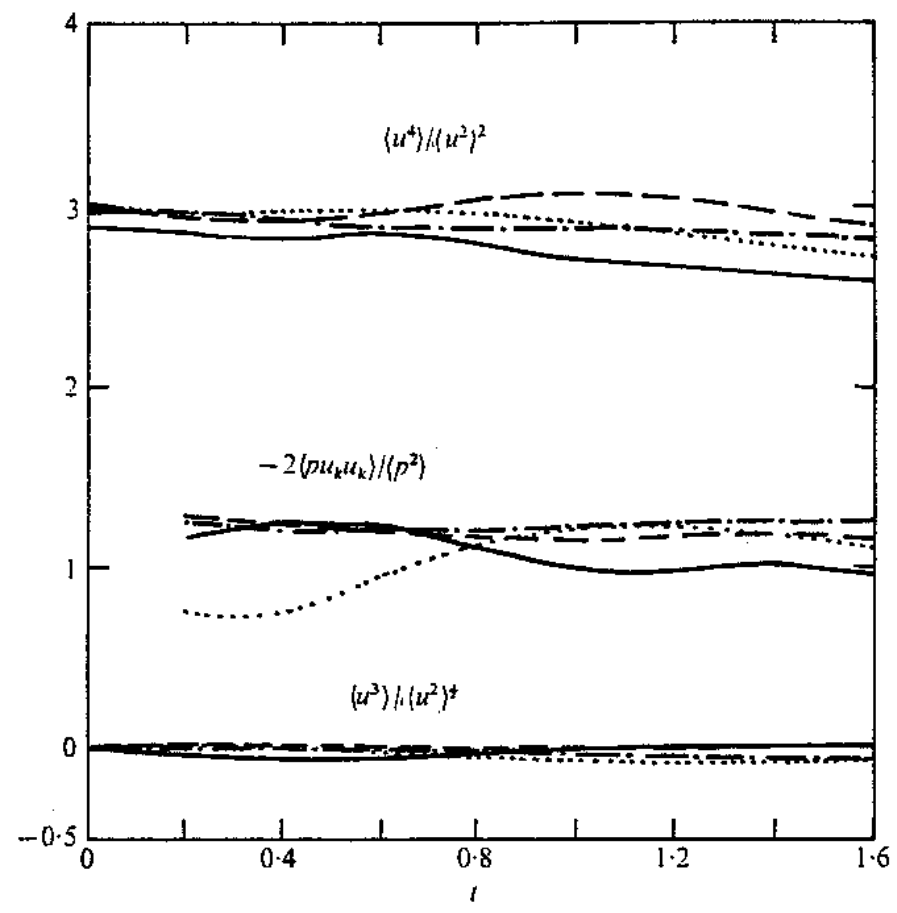

FrguRe 16. One-point fourth-order correlations vs. time. ,$- I 1 ;---, I 2 ;-.-, I 3 ; \cdots \cdots, I 4$. 


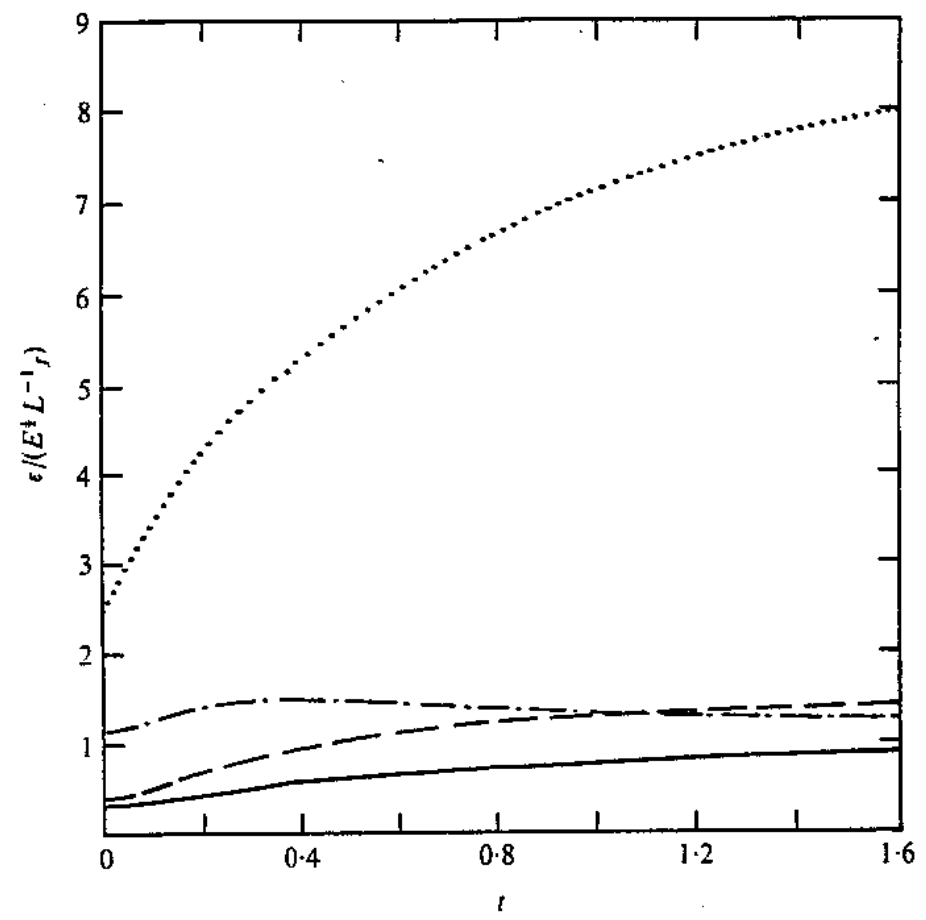

FIGURE 17. Normalized dissipation v8. time.,$- I 1 ;---, I 2 ;-\cdot-, I 3 ; \cdots \cdots, I 4$.

should be zero for isotropic turbulence. All these estimates are confirmed by the numerical results shown in figure 16.

\subsection{Dissipation rate}

According to the well-accepted inertial-subrange theory (e.g. Batchelor 1959, §6.1) the ratio $\epsilon L_{f} / E^{\frac{3}{2}}\left(E=\frac{1}{2}\langle\mathbf{u} \cdot \mathbf{u}\rangle\right)$ should be a number of order unity and nearly constant. This is not true for low Reynolds numbers, as can be seen from figure 17, especially for run I4. Similar results have been found by Kraichnan (1964), Orszag \& Patterson (1972 $a$ ) and Herring (1973). The inertial-subrange theory assumes that the dissipation rate is mainly determined by the energy transfer effected by the inertial terms. However, at low Reynolds numbers the direct viscous dissipation at low wavenumbers (characterized by $L_{f}$ ) becomes important too. Following Rotta (1951), we therefore propose the relationship

$$
\epsilon=B_{1} \nu \frac{E}{L_{f}^{2}}+B_{2} \frac{E^{2}}{L_{f}}
$$

where $B_{1}$ and $B_{2}$ are numbers which should be only slightly dependent upon the Reynolds number and the initial values used. If the Reynolds number $R e_{L}=\left(\frac{2}{3}\right)^{\frac{1}{2}} E \frac{1}{2} L_{f} / \nu$ is large, the first term becomes negligible. If this Reynolds number approaches zero, the second term becomes zero. Assuming $R_{l}^{l}(r)$ to be given by (12), we get

$$
L_{f}^{2}=\frac{1}{2} \pi \lambda^{2}
$$

and therefore

$$
\epsilon=10 \nu E / \lambda_{2}=5 \pi \nu E / L_{f}^{2},
$$

which corresponds to

$$
B_{1}=5 \pi \approx 15 \cdot 7 \text {. }
$$




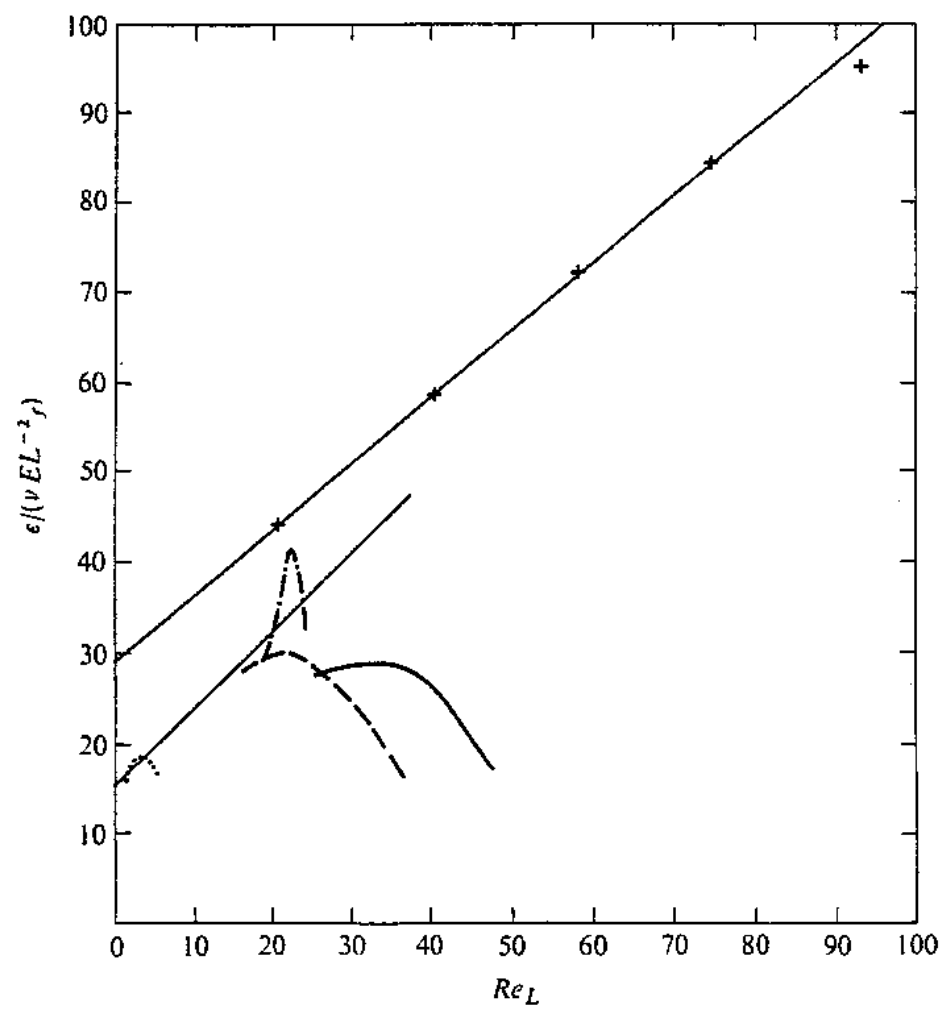

Frauke 18. Normalized dissipation vs. Reynolds number.,$- I 1 ;---, I 2 ;-., I 3$; $\cdots \cdots, I 4 ;+$, Herring (1973); -

The ratio

$$
\epsilon / \nu E L_{f}^{2} \approx B_{1}+\left(\frac{3}{2}\right)^{\frac{1}{2}} B_{2} R e_{L}
$$

is plotted $v s . R e_{L}$ in figure 18. Here time decreases as $R e_{L}$ increases. All curves show some increase in the normalized dissipation rate owing to vortex-line stretching in the initial phase. The right end point of each curve corresponds to time $t=0$. Here the dissipation results from purely viscous effects and when normalized should equal $B_{1}$. We find that these points do agree, according to (20), for runs $I 1, I 2$ and $I 4$, where the initial spectrum is close to the self-similar form for $R e_{\lambda} \rightarrow 0$. The departure for run $I 3$ is a consequence of the different initial energy spectrum. After some time a maximum in the normalized dissipation is reached owing to the inertial energy transfer; the dissipation then decreases again as the inertial transfer is damped by the viscous effects. The appropriate value of $B_{2}$ is determined by the slope of the line which is tangential to the experimental curves after the initial development of the flow and passes through $\left(0, B_{1}\right)$. Case $I 1$ has insufficient data and case $I 3$ differs owing to the energy spectrum. From case $I 4$ and especially case $I 3$ we find $B_{2} \approx 0.7$, which is in reasonable agreement with the experimental results $\left(B_{2} \approx 0.9 \pm 0 \cdot 2\right)$ reported by Batchelor (1959, figure 6.1, constant $\left.A \equiv B_{2}\left(\frac{3}{2}\right)^{\frac{1}{2}}\right)$. Herring's (1973) results do confirm the assumption in (18) but give somewhat different values for $B_{1}$ and $B_{2}$, which seems to be a consequence of the different initial spectrum. Herring used

$$
\hat{E}(k, 0)=\lambda^{2}\left(1+2 \frac{1}{2} k \lambda\right) \exp \left(-2 \frac{1}{2} k \lambda\right) \text {. }
$$

The crosses in figure 18 mark the values which are computed some time after the self-similar state has been reached. 
The principal difference between the initial energy spectra (9) and (10) becomes obvious if we determine the value of $n$ in an assumed power law $E \sim t^{-n}$. This exponent is deduced from figure 19, which depicts the decay of energy as a function of time on logarithmic scales. The slopes at the final times correspond to $n=2 \cdot 3,1 \cdot 6,1 \cdot 0$ and 1.7 for cases $I 1, I 2, I 3$ and $I 4$ respectively. Actually the slopes are still varying at the final time 1.6 used up to now. Therefore additional data are included for case $I 1$ up to $t_{\max }=15$. From these we expect final decay laws with $n \approx 2.5$ for cases using the initial spectrum (9) and $n \approx 1.0$ for (10). These results are not discordant with theories (Leith 1967) and experimental data: Uberoi (1963) and Comte-Bellot \& Corrsin (1966), for example, found $n=1 \cdot 1 \pm 0.2$ for high Reynolds numbers; Batchelor \& Townsend (1948) and Bennett (1976) measured $n \approx 2.5$ for Reynolds numbers which are of comparable size to those of the numerical study. This shows that the decay law is not directly dependent on the Reynolds number rather than on the type of initial spectrum, which in turn might depend on the Reynolds number.

\subsection{Dissipation tensor for small departures from isotropy}

Although runs $I 1-I 4$ were designed to be isotropic they are not completely so. Thus we get non-zero values for the off-diagonal components of the energy and dissipation tensors $E_{i j}$ and $\epsilon_{i j}$, and the diagonal components are not generally equal. Here

$$
E_{i j}=\left\langle u_{i} u_{j}\right\rangle, \quad E=\frac{1}{2} E_{i i}, \quad \epsilon_{i j}=2 \nu\left\langle\frac{\partial u_{i}}{\partial x_{k}} \frac{\partial u_{j}}{\partial x_{k}}\right\rangle, \quad \epsilon=\frac{1}{2} \epsilon_{k k} .
$$

We use the convention that repeated subscripts are summed from one to three. If the Reynolds number is large enough the dissipation tensor should be isotropic even if the energy tensor is anisotropic. For very low Reynolds numbers these tensors are directly proportional to each other. Following Corrsin (1973) and Naot, Shavit \& Wolfshtein $(1973,1974)$, we therefore propose the following model:

$$
\epsilon_{i j} \approx \epsilon_{i j}^{m} \equiv \frac{2}{3} \epsilon\left[\delta_{i j}(1-d) \div\left(3 E_{i j} / E_{k k}\right) d\right],
$$

where $d$ is a function of $R e_{\lambda}$ and possibly other parameters and $\delta_{i j}$ is the Kronecker delta. As $d$ should be 1 for $R e_{\lambda}=0$ and 0 for $R e_{\lambda}=\infty$, we propose

$$
d=1 /\left(1+\alpha R e_{\lambda}\right),
$$

where $\alpha$ is an empirical constant.

Using the present runs we determine $d$ for each time step from a least-squares fit, so that

$$
F(d)=\sum_{i=1}^{3} \sum_{j=1}^{\mathrm{a}}\left(\epsilon_{i f}-\epsilon_{i j}^{m}(d)\right)^{2}=\text { minimum. }
$$

The resultant values $d(t)$ are plotted $v s . R e_{\lambda}(t)$ in figure 20 , where time decreases as $R e_{\lambda}$ increases. For $t=0$ the values of $d$ are close to 1 , which means that no local isotropy exists initially. After some time the anisotropy at the high wavenumbers (where most of the dissipation is concentrated) is diminished, whereas the anisotropy at low wavenumbers (where most of the energy is concentrated) is not reduced to the same degree. Therefore $d$ is diminished and the final value $d\left(R e_{\lambda}\right)$ should be determined by these smaller values. The line corresponding to (23) with $\alpha=0.1$ seems to be an appropriate fit for this final state. 


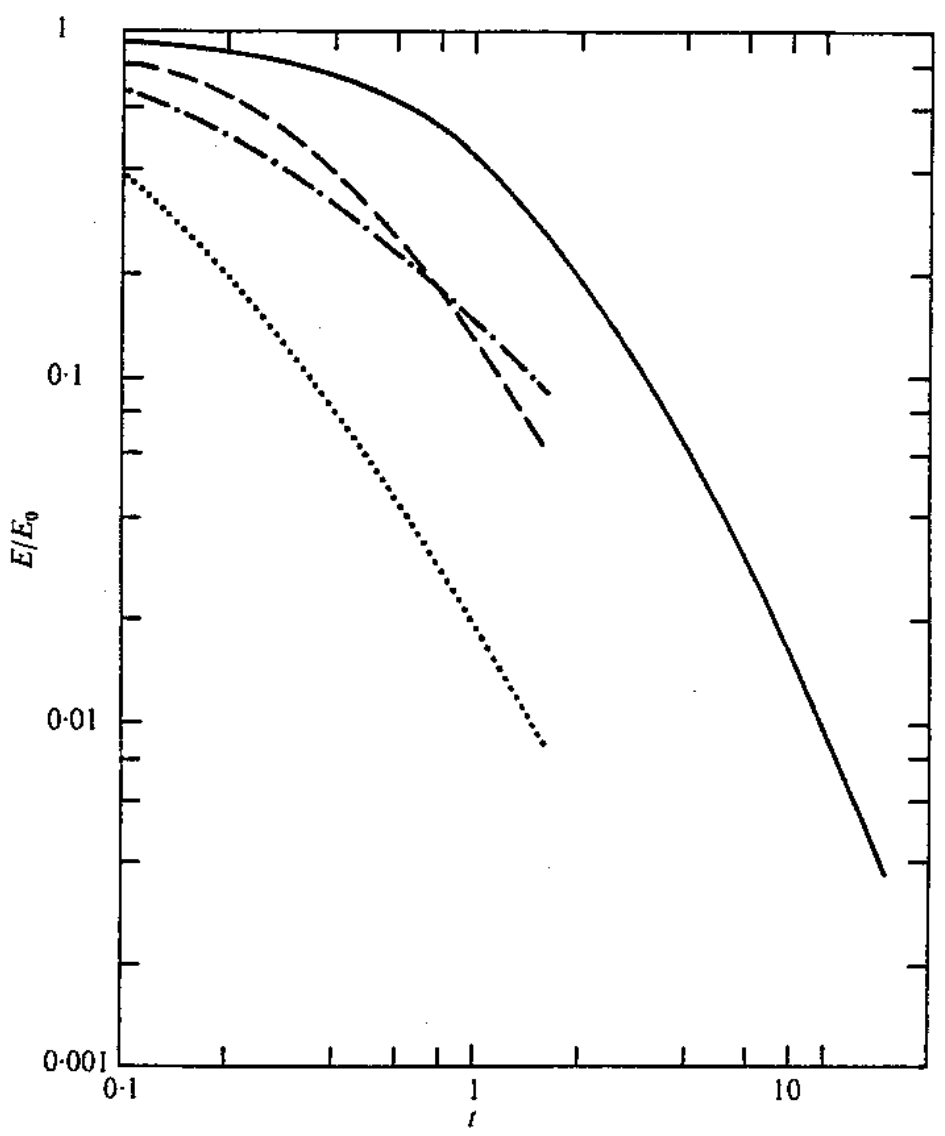

FIGURe 19. Energy normalized by $E_{0}=E(0)$ vs. time showing a final power-law decay $E \sim t^{-n}$. -,$I 1 ;---, I 2 ;-\cdot-, I 3 ; \cdots \cdots, I 4$.

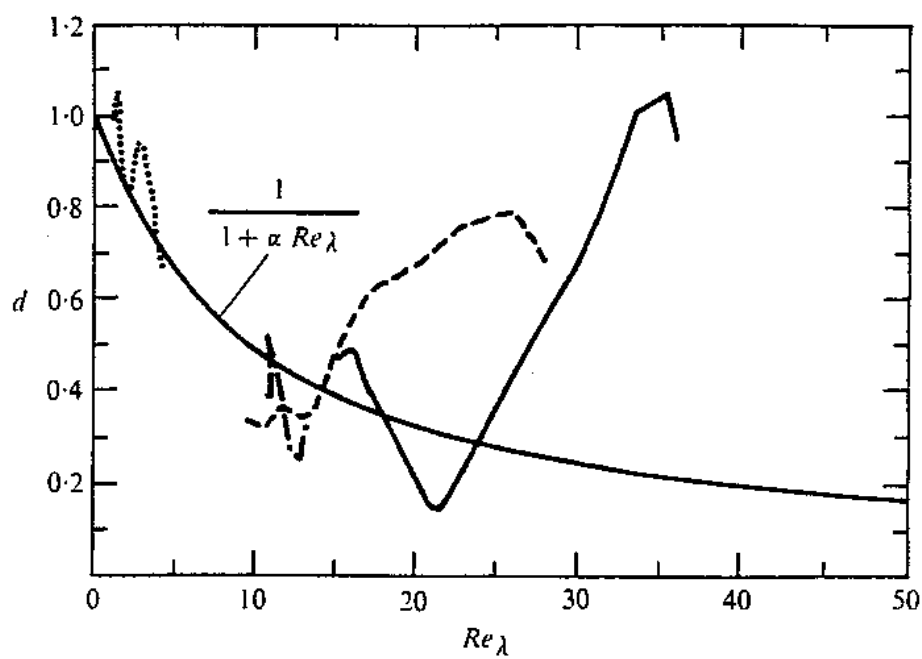

Frgure 20. Parameter $d$ characterizing the departure from local isotropy for the dissipation tensor vs. Reynolds number. $\alpha=0.1 . \longrightarrow, I 1 ;---, I 2 ; \ldots . \ldots, I 3 ; \cdots \cdots, I 4$. 


\section{Summary}

The spectral method of Orszag \& Patterson $(1972 a, b)$ has been extended to simulate the fluctuating pressure field in homogeneous turbulence. The resultant statistics have been evaluated for decaying isotropic turbulence. The Reynolds number $R e_{\lambda}$ varies between 1 and 36. Different initial energy spectra have been assumed. An energy spectrum of the form $k \exp (-k)$ is close to a self-similar shape and results in correlation functions (figure 13a) and decay laws (figure 19) which agree reasonably well with experimental results for even rather large Reynolds numbers. The errors due to a truncated Fourier-series representation are, however, considerably smaller for an energy spectrum of the form $k^{4} \exp \left(-k^{2}\right)$. The decay law for this initial spectrum corresponds well to those found experimentally for small Reynolds numbers. For the ratios $\left\langle p^{2}\right\rangle / v^{2}, \lambda_{p} / \lambda$ and $L_{p} / L_{f}$, the shape of the initial spectrum has been found to be of small importance.

We found the r.m.s. value of the pressure fluctuations normalized by the meansquare velocity to be about 0.9 at $R e_{\lambda} \approx 30$ and about 1.0 at $R e_{\lambda} \approx 0$. The microscale of the pressure fluctuations is about 0.7 times the microscale of the velocity fluctuations; the integral length scales of the pressure and velocity fluctuations have a ratio of about 0.55 . All these results agree well with experimental and theoretical findings. From the scatter of the numerical results around smoothed curves one can estimate (see figures 9 and 11) that the statistical errors are of the order of $10 \%$ for these quantities. This suggests that the numerical experiments give somewhat more accurate results than known laboratory experiments where the scatter is at least of the order of $30 \%$ for these quantities (see, for example, figure 12). The statistical errors in the numerical simulations are due to the finite amount of data from which we computed mean values. Reduction of these errors would require the expensive generation of a larger ensemble and this has not been feasible for this study.

The theoretical and experimental results were all based on the assumption of normality of fourth-order velocity correlations. The normality assumption has been verified for these simulations; the departure lies within the limits of statistical error. This result, which is surely very helpful in estimating pressure statistics, cannot be used, however, to close turbulence models for the first, second and third velocity moments: it has been shown by Ogura (1963) that use of the normality assumption can lead to non-realizable flows (e.g. negative energy). The assumption

$$
R_{l}^{l}(r) \approx v^{2} \exp \left(-r^{2} / 2 \lambda^{2}\right)
$$

has been confirmed for small Reynolds numbers. This assumption allows analytical solutions for the different correlations investigated here. For the correlation between pressure and second-order velocity products, this analytical solution is given in (14)-(17).

Owing to the small number of modes represented by the finite Fourier series at small wavenumbers, the initial conditions are not completely isotropic here. This departure from isotropy becomes dominant during the final period of the decay. It was possible, therefore, to study the total rate of viscous dissipation as well as its tensorial distribution. For both, simple empirical models have been deduced which can be used in practical turbulence model integrations.

The results presented here show the applicability of the method to low Reynolds 
number simulations of turbulent pressure and velocity fields. Anisotropic homogeneous turbulence is studied in the companion paper.

We are very grateful to J. R. Herring for many helpful discussions and for making available the data used in figure 17. We also thank T.J. Wright, who developed the advanced computer graphics routines used here and assisted in their application, and M. Frydrych, who adapted the isosurface plotting routine to the data management used in the spectral code. Also, we are very grateful to the referees for several constructive suggestions. This work was done while U. Schumann was with the Advanced Study Program at the National Center for Atmospheric Research, which is sponsored by the National Science Foundation.

\section{REFERENCES}

ARNDT, R. E. A. \& Nrusin, A.W. 1971 On the measurement of fluctuating pressure in the mixing zone of a round jet. A.S.M.E. Paper no. 71-FE-31.

Batohelor, G. K. 1951 Pressure fluctuations in isotropic turbulence. Proc. Oamb. Phil. Soc. 47, 359-374.

BATOHELOR, G. K. 1959 The Theory of Homogeneous Turbulence. Cambridge University Press.

Batcheror, G. K. \& Townsend, A. A. 1948 Decay of turbulence in the final period. Proc. Roy. Soc. A 194, 527-543.

BENNETT, J. C. 1976 Measurements of grid-generated, nearly isotropic turbulence at small Reynolds numbers. Thesis, Johns Hopkins University.

Comete-Beiclot, G. \& Corrsin, S. 1966 The use of a contraction to improve the isotropy of grid-generated turbulence. J. Fluid Mech. 25, 657-682.

CoRzsIn, S. 1973 Comment on 'Transport equations in turbulence'. Phys. Fluids 16, 157-158.

DEARDORFP, J.W. 1970 A numerical study of three-dimensional turbulent channel flow at large Reynolds numbers. J. Fluid Mech. 41, 453-480.

DEARDORFF, J.W. 1974 Three-dimensional numerical study of turbulence in an entraining mixed leyer. Boundary-Layer Met. 7, 199-226.

Euxior, J.A. 1972 Microscale pressure fluctuations measured within the lower atmospheric boundary layer. J. Fluid Mech. 53, 351-383.

FrENKTrL, F. N. \& KTIBBANoTr, P. S. 1967 High-order correlations in a turbulent field. Phys. Filuids 10, 507-520.

FucHs, H. V. 1972 Space correlations of the fluctuating pressure in subsonic turbulent jets. $J$. Sound Vib. 23, 77-99.

Hamperstiey, R. J. \& Jonfes, B. G. 1975 Turbulent pressure field in a co-annular jet. A.I.A.A. Paper no. 75-95.

Heismanara, W. 1948 Zur statistischen Theorie der Turbulenz. Z. Phys. 124, 628-657.

HzRRnNG, J. R. 1973 Statistical turbulence theory and turbulence phenomenology. In Proc. Langley Working Conf. Free Turbulent Shear Flows, Langley Res. Center, Langley, Virginia. N.A.S.A. Special Paper no. 321, pp. 41-66.

KADLEC, J. \& APPEIT, K. D. 1970 Flow induced rod vibrations of fast reactor aubassemblies. Nucl. Engng Design 14, 136-150.

KoBastr, Y. 1957 Measurements of pressure fluctuation in the wake of cylinder. J. Phys. Soc. Japan 12, 533-543.

Kratoman, R. H. 1956 a Pressure field within homogeneous anisotropic turbulence. J. Acoust. Soc. $A m .28,64-72$.

KraichnaN, R. H. $1956 b$ Pressure fluctuationg in turbulent flow over a flat plate. J. Acoust. Soc. Am. 28, 378-390.

KratchinaN, R. H. 1964 Decay of isotropic turbulence in the direct-interaction approximation. Phys. Fluids 7, 1030-1048. 
KणO, A. Y.-S. \& CoRRsn, S. 1971 Experiments on internal intermittency and fine-structure distribution functions in fully turbulent fluid. J. Fluid Mech. 50, 285-319.

LEITH, C. E. 1967 Diffusion approximation to inertial energy transfer in igotropic turbulence. Phys. Fluids 10, 1409-1416.

Lmazer, D. N. 1951 Numerical results for pressure-velocity correlations. Proc. Nat. Acad. Sci. US.A 37, 230-233.

NAOT, D., SHAVTr, A. \& WoLfSHTEI, M. 1973 Two-point correlation model and the redistribution of Reynolds stresses. Phys. Fluids 16, 738-743.

NaOT, D., Shavit, A. \& Wolfshiten, M. 1974 Numerical calculation of Reynolds stresbes in a Bquare duct with secondary flow. Wärme Stoffübertragung 7, 151-161.

OGURA, Y. 1963 A consequence of the zero-fourth-cumulant approximation in the decay of isotropic turbulence. J. Fluid Mech. 16, 33-40.

OGURA, Y. \& MTYAKoDA, K. 1954 Note on the pressure fluctuations in isotropic turbulence. J. Met. Soc. Japan 32, 160-166.

Orszag, S. A. 1971 Numerical simulation of incompressible flow within simple boundaries. I. Galerkin (spectral) representations. Stud. Appl. Math. 50, 293-327.

Orszac, S. A. \& KruskaI, M. D. 1968 Formulation of the theory of turbulence. Phys. Fluids $11,43-60$.

OrszaG, S. A. \& Patrierson, G. S. 1972 a Numerical simulation of turbulence. In Statistical Models and Turbulence, pp. 127-147. Springer.

Orszac, S. A. \& PatTerson, G.S. $1972 b$ Numerical simulation of three-dimensional homogeneous isotropic turbulence. Phys. Rev. Lett. 28, 76-79.

Panton, R. L. \& Linefaragr, J. H. 1974 Wall pressure spectra calculations for equilibrium boundary layers. $J$. Fluid Mech. 65, 261-287.

PatTrason, G.S. \& Orszae, S. A. 1971 Spectral calculations of isotropic turbulence: efficient removal of aliasing interactions. Phys. Fluids 14, 2538-2541.

RIBNER, H. P. 1964 The generation of kound by turbulent jets. Adv. Appl. Mech. 8, 103-187.

RIIEY, J.J. \& Patmerson, G.S. 1974 Diffusion experiments with numerically integrated isotropic turbulence. Phys. F'luids 17, 292-297.

Rorra, J. 1951 Statistische Theorie nichthomogener Turbulenz. Z. Phys. 129, 547-572.

Sorromank, U. 1975 a Subgrid scale model for finite difference simulations of turbulent flows in plane channels and annuli. J. Comp. Phys. 18, 376-404.

SoHumanN, U. $1975 b$ Numerical investigation of the wall pressure fluctuations in channel flowg. Nucl. Engng Design 32, 37-46.

Spencer, B. W. \& Jones, B. G. 1971 Statistical investigation of pressure and velocity felds in the turbulent two-stream mixing layer. A.I.A.A. Paper no. 71-613.

Tavouraris, S. 1977 Pregentation to Turbulence Conf. N.C.A.R.

TAYLOR, G. I. 1935 Statistical theory of turbulence. IV. Diffusion in a turbulent eir stream. Proc. Roy. Soc. A 151, 465-478.

UBEROI, M. S. 1953 Quadruple velocity correlations and pressure fluctuations in isotropic turbulence. J. Aero. Sci. 20, 197-204.

UBezoI, M. S. 1954 Correlations involving pressure fluctuations in homogeneous turbulence. N.A.C.A. Tech. Note no. 3116.

Urzeror, M. S. 1963 Energy trangfer in isotropic turbulence. Phys. Fluids 6, 1048-1056.

UBERor, M. S. \& CoRRSIN, S. 1953 Diffusion of heat frorn a line source in isotropic turbulence. N.A.C.A. Rep. no. 1142.

Wrimarth, W. W. 1975 Pressure fluctuations beneath turbulent boundary layers. Ann. Rev. Fluid Mech. 7, 13-38. 